\begin{tabular}{|c|c|c|c|c|c|}
\hline MUNIBE Antropologia-Arkeologia & $n^{\circ} 68$ & $49-72$ & DONOSTIA & 2017 & ISSN 1132-2217 • elSSN 2172-4555 \\
\hline
\end{tabular}

\title{
Las cadenas operativas líticas de El Barandiallu (Asturias, España): adaptación y variabilidad tecnológica en el contexto del Musteriense cantábrico
}

\author{
Chaînes- opératoires of El Barandiallu (Asturias, Spain): adaptation \\ and technological variation in the context of the Cantabrian Mousterian
}

PALABRAS CLAVES: MTA, Yacimiento al aire libre, Paleolítico Medio, Tafonomía, Tecnología lítica.

GAKO-HITZAK: MTA, aire zabaleko aztarnategia, Erdi-paleolitoa, tafonomia, teknologia litikoa.

KEY WORDS: MAT, Open air site, Middle Palaeolithic, Taphonomy, Lithic Technology.

\section{David ÁLVAREZ-ALONSO(1)}

\section{RESUMEN}

En este trabajo se presenta el estudio completo del conjunto lítico musteriense de El Barandiallu, con más de 1400 piezas, formado por materiales recogidos en superficie, recuperados en 1988 y 1989, y por materiales procedentes de una excavación arqueológica, realizada en el año 2012. Este conjunto lítico se sitúa como uno de los más interesantes entre los localizados al aire libre en la región cantábrica. A la hora de analizar el conjunto e interpretar la ocupación humana que ha dado lugar al mismo, se plantean interesantes debates sobre la diferencia de los yacimientos musterienses en cueva y al aire libre, en cuanto a su composición tipológica y sus características tecnológicas, que no solo nos plantea cuestiones funcionales, sino también nos acerca a un viejo e interesante debate sobre la caracterización tecnotipológica del Musteriense en la región, sus relaciones con el mundo pirenaico y aquitano y la existencia o no de conjuntos equiparables, coetáneos y parcialmente compatibles con el MTA.

\section{LABURPENA}

El Barandialluko Moustier aldiko multzo litikoko azterketa osoa azaltzen da lan honetan. Multzoak 1400 pieza baino gehiago ditu eta gainazalean jasotako materialek (1988 eta 1989 artean berreskuratutakoak) eta 2012an egindako indusketa arkeologikoan jasotako materialek osatzen dituzte. Multzo litiko hori Kantabria eskualdean aire zabalean topatutako interesgarrienetako bat dela esan dezakegu. Multzoa aztertzeko eta bertako gizakiaren okupazioa interpretatzeko garaian, kobazuloko eta aire zabaleko Moustier aldiko aztarnategien arteko ezberdintasunari buruzko eztabaida interesgarriak sortu dira bertako osaera tipologikoa eta ezaugarri teknologikoak direla eta. Horiek guztiek gai funtzionalak mahaigaineratzeaz gain, gai hauek oinarri dituen eztabaida zahar eta interesgarri batera gerturatu gaituzte: eskualdean Moustier aldiak izan zuen karakterizazio teknotipologikoa, Pirinioetako eta Akitaniako munduarekin izandako harremanak edo alderagarriak, garaikideak eta MTArekin (Acheul tradizioko Moustier aldia) partzialki alderagarriak diren multzoak izatea (edo halakorik ez izatea).

\section{ABSTRACT}

More than 1400 lithics, which is formed by surface findings recovered between 1988 and 1989, as well as materials collected in an archaeological excavation developed in 2012. This Mousterian lithic assemblage stands as one of the most interesting among open air sites in the Cantabrian region. Likewise, it is the only case studied in the north of the Iberian Peninsula of this type of assemblage in which a taphonomical process caused by modern and contemporaneous agricultural action has been identified.

When analysing the assemblage and interpreting its human occupation, interesting debates about the difference between Mousterian openair and cave sites, their typological composition and their technological characteristics -which not only consider functional issues, but also bringing us closer to an old and interesting debate about the techno-typological characterization of the Mousterian in the area, its relationships with the Pyrenees and Aquitaine and the existence or nonexistence of comparable, contemporary and partially compatible with the Mousterian of Acheulean Tradition (MAT)-, are considered.

This settlement seems to adjust to the characteristic of a temporary occupation, with a high degree of closeness in the procurement of resources (most of the identified raw materials are local) and in the Chaîne opératoires developed,although it has been possible to identify a different a different processing in relation to the Chaîne opératoire, both at a raw material level and a technological level. Furthermore, it has been also possible to observe the existence of complete and amortized Chaîne opératoires that underpin the abovementioned immediacy, while at the same time the transportation of lithics from other relative close (macrotools) and further away places (pieces of flint) has been identified.

Thus, we can stay that in the case of El Barandiallu the technological variability perceived in this assemblage may be the consequence of the type of functionalities and circumstances of the open-air human occupation, in relation to the occupations in caves, which are more recurrent and less ephemeral.

\footnotetext{
(1) Departamento de Prehistoria y Arqueología, UNED-Asturias. Avda. Jardín Botánico 1345 (Calle interior) 33203 Gijón, Asturias. dalvarez@gijon. uned.es
} 
When looking for a parallel to El Barandiallu, it is essential to look at the French southwest, where there are various Mousterian sites ranging between the final MIS4 and the MIS3. In these French sites, Levallois and discoid knapping with a small percentage of handaxes (in non-local flint) and, in the least of cases, cleavers made in quartzites or local ophites were documented. We are especially interested in sites such as La Prissé, Chemin de Jupiter or Latrote, all of them open-air sites, where quartzite cleavers and some handaxes made from a distant source were found, in a context where the raw material are essentially local.

On the other hand, it is possible that the relative chronology of El Barandiallu, with a techno-typological characterization compatible with the Aquitanian MAT contexts, fits with these French sites. Likewise, we can establish a link with assemblages documented in caves such as Abauntz h; La Viña XIII basal, which has been identified as a final Mousterian level with cleaver, El Castillo 20; or the mousterian series with cleavers from Cueva Morín. These parallels refer to a relatively recent context within the Mousterian at El Barandiallu, which is placed in any case in a contemporaneous or posterior to MIS4 context.

\section{1.- INTRODUCCIÓN}

Las ocupaciones musterienses documentadas en la Región Cantábrica se circunscriben, en su gran mayoría, a depósitos en cueva que han venido siendo excavados desde hace décadas, como por ejemplo: El Conde, La Viña, El Sidrón o La Güelga en Asturias; Esquilléu, Covalejos, Morín, El Pendo o El Castillo, en Cantabria; Axlor, Lezetxiki, Amalda o Arrillor en el País Vasco, por citar algunos de los más significativos (Altuna et al., 1990; Arbizu et al., 2009; Arrizabalaga, 2006; Arrizabalaga et al., 2005; Baena y Carrión, 2014; Cabrera y Bernaldo De Quirós, 2000; Fortea, 1999; González-Echegaray y Freeman, 1978; González-Urquijo et al., 2006; Maíllo et al,. 2014; Martín et al., 2006; Menéndez et al., 2009, 2014; Montes, 2014; Rasilla et al., 2011; Sáenz de Buruaga et al., 1999). De este modo, se tiene bastante información sobre el poblamiento neandertal del norte peninsular, a partir de numerosos registros en cuevas o abrigos. Por el contrario, los yacimientos al aire libre han quedado relegados en la investigación cantábrica debido, principalmente, a la abundancia y accesibilidad de las cuevas en la región, o por las dificultades para la conservación y prospección de los sitios paleolíticos al aire libre (Arrizabalaga et al., 2015). A pesar de esto, algunos pocos yacimientos del Paleolítico medio al aire libre han sido excavados en las últimas décadas, como El Habario, El Hondal y La Verde (Cantabria), Mendieta (Vizcaya), o más recientemente El Barandiallu, en Asturias y Aranbaltza en Vizcaya (Álvarez-Alonso, 2013; Carrión y Baena, 2005; Montes, 1999, 2003; Ríos, 2013; Ríos et al., 2010), mientras que otros yacimientos clásicos al aire libre, como Bañugues, han sido recientemente reclasificados dentro del Paleolítico medio (Álvarez-Alonso, 2014; Álvarez-Alonso et al., 2014a). Estos lugares, al margen de encuadrarse en un amplio espectro cronológico situado entre el MIS 5e y el MIS 3, también nos muestran, por sus diferentes características, la existencia de distintas tipologías de yacimientos, dejando entrever una relativa variedad en la estructuración de los modelos tecnoeconómico y de adaptación territorial por parte de los grupos neandertales en el espacio cantábrico. Como consecuencia, queda patente que durante el Musteriense parece existir cierta variedad funcional y habitacional en cuanto a la gestión y organización del espacio socio-económico por parte de los neandertales cantábricos, a tenor de lo observado por los registros en cueva y al aire libre.

Si bien los datos existentes son limitados, en lo que respecta a los yacimientos en cueva se suele contar con restos de macro y microfauna, análisis polínicos, antracológicos, y cronoestratigráficos más o menos exhaustivos y de gran importancia para obtener información paleoclimática, que además incorporan dataciones fundamentalmente obtenidas por métodos radiocarbónicos. Estas dataciones se corresponden, en su mayoría, con niveles pertenecientes a las últimas fases del Musteriense, dentro del MIS 3, mientras que tan solo una mínima parte pertenece a contextos del Paleolítico medio situados por encima del MIS 4 (que principalmente se encuadran entre los MIS 7-5). Así es como sucede con los niveles más antiguos de Lezetxiki, Arlanpe, Covalejos y El Castillo o en la zona A de La Garma (Álvarez-Alonso y Arrizabalaga, 2012; Bischoff et al., 1992; Falguerès et al., 2006; Montes, 2003; Ríos et al., 2015; Tapia, 2010), o incluso en Cueva Corazón, en la vertiente sur de la cordillera (Díez et al., 2011), existiendo así en esta región, una manifiesta carencia de dataciones para contextos situados en el MIS 4 (Álvarez-Alonso, 2014). A pesar de esta situación, la presencia de neandertales en la cornisa cantábrica parece ser continua, al menos desde el MIS 6, con gran representación de secuencias musterienses desde el MIS 5e hasta el MIS 3b (Álvarez-Alonso, 2012, 2014; Sánchez y Díez, 2015).

En lo que a la posibilidad de recuperación de restos variados se refiere, no sucede lo mismo con los yacimientos al aire libre, donde las posibilidades de contextualización son menores con respecto a los yacimientos en cueva (Arrizabalaga et al., 2015). En este caso, la industria lítica suele ser la única herramienta (o una de las pocas) que posibilita un acercamiento crono-cultural que nos permite iniciar un intento de contextualización con el resto de evidencias musterienses localizadas a lo largo de la región cantábrica, como así sucede en el caso de El Barandiallu. No obstante, los yacimientos al aire libre cantábricos presentan ciertas peculiaridades de índole

\footnotetext{
${ }^{1}$ Doce de ellas se recogieron en superficie en el entorno de la excavación.

2 La concentración del material es elevada, por lo que consideramos que se trata de un emplazamiento primario (en lo que respecta a la localización, no al contexto estratigráfico), bien localizado, aunque con una importante alteración en su estructura y relación arqueológica interna.
} 
geoarqueológica, sobre todo en lo que se refiere a los yacimientos en contextos edáficos, como es el caso que presentamos (Álvarez-Alonso, 2015; Arrizabalaga et al., 2015) y que, en definitiva, resulta ser la principal limitación con respecto a los sitios en cueva.

\section{2.- LOCALIZACIÓN Y CONTEXTO GEOLÓGICO}

El Barandiallu se localiza en La Vega (Villardebeyo, Llanera, Asturias) en el valle del río Frade/Aboño, presentando una orientación O-E en la zona de estudio, a una altitud s.n.m de 126-128 m, siendo sus coordenadas UTM: X.272235, Y.4816475 (Huso 30; ETRS 89). La zona presenta un relieve dominado por suaves lomas y pequeñas elevaciones, donde la altitud mayor la alcanza la cima del Picu Santufirme (439 m), mientras que la más baja es el curso del río Aboño a su paso por La
Vega (100 m) (Fig.1). Precisamente, en el Santufirme y su prolongación se encuentra la divisoria de aguas entre los cauces que drenan directamente al mar Cantábrico, como el Aboño, y los que lo hacen hacia el sur, hacia la depresión central asturiana por la que discurren el Nora y el Noreña, que pertenecen ya a la cuenca hidrográfica del río Nalón. Estamos, por tanto, en lo que se considera ya la depresión prelitoral de la región central asturiana. A su vez, el valle del Aboño está excavado sobre materiales de edad triásica, donde el cauce discurre fundamentalmente entre arcillas del Keuper, destacando en las laderas cercanas la presencia de conglomerados cuarcíticos del Buntsandstein y del Jurásico inferior (Fig.2).

El yacimiento se sitúa en la parte superior de un depósito de ladera que inicialmente fue interpretado como un glacis-terraza (Estrada y Jordá, 2004), circunstancia

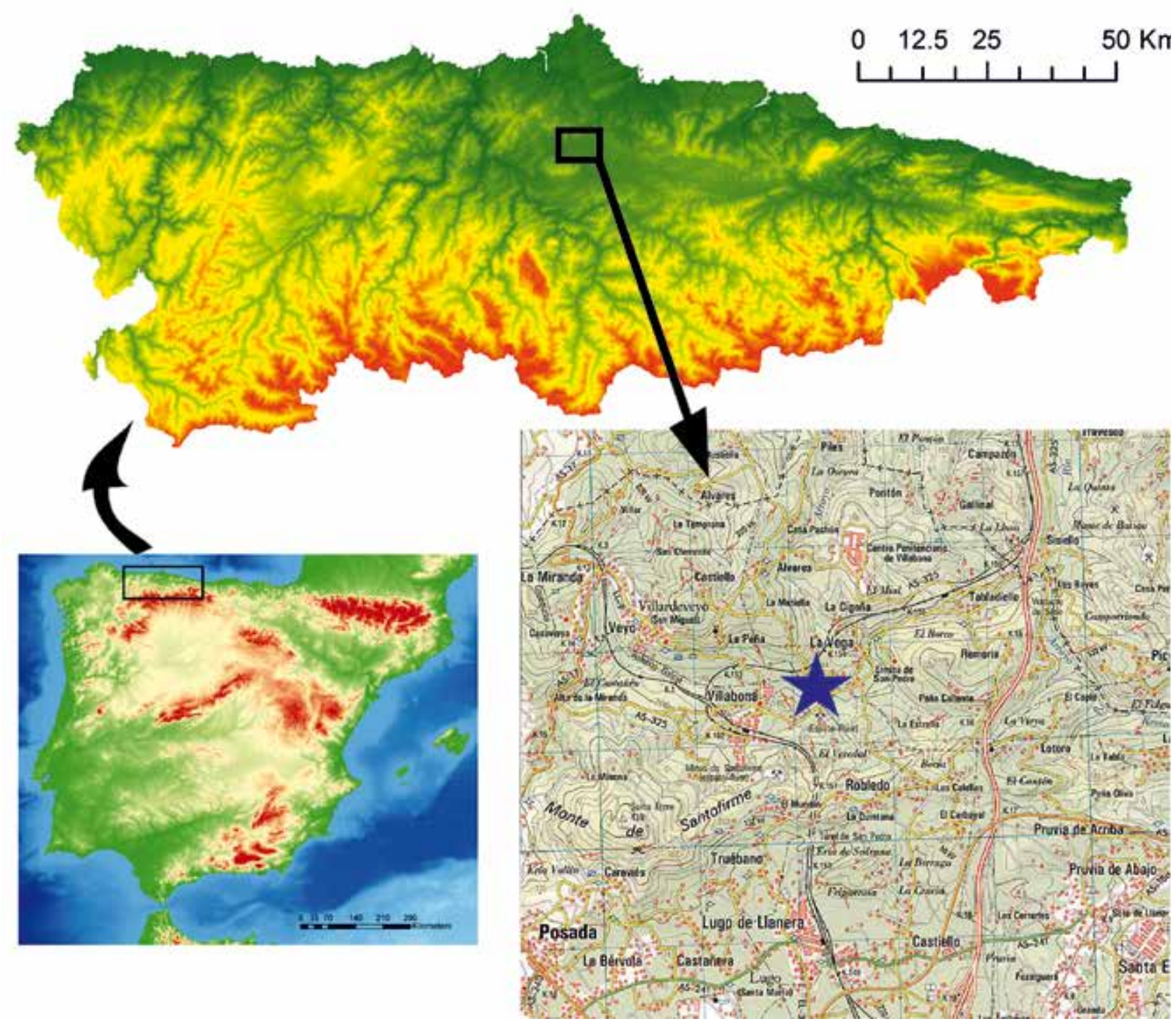

Fig. 1. Localización del yacimiento de El Barandiallu / Barandiallu site location. 


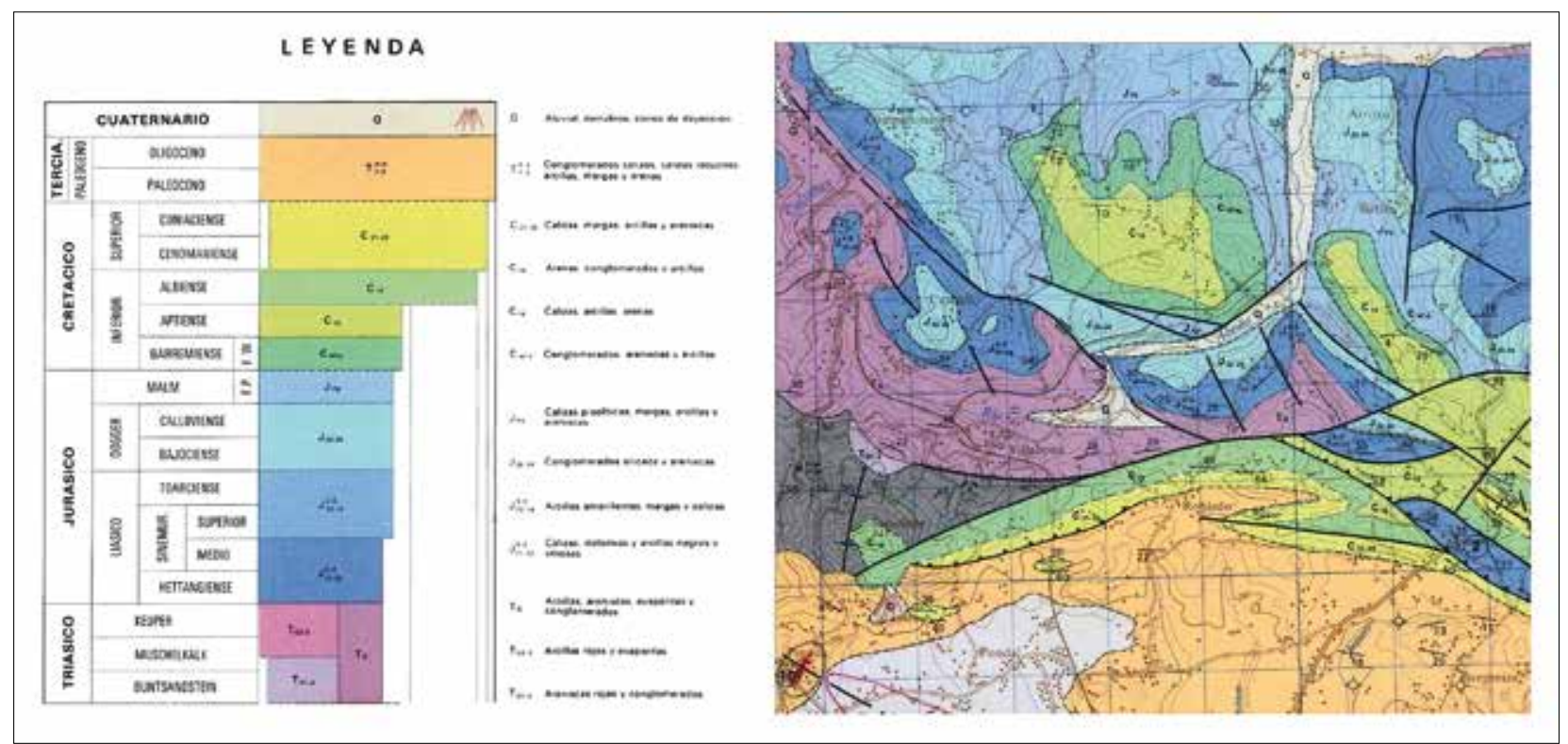

Fig. 2. Mapa geológico de contexto para la zona de estudio (modificado, IGME) / Geological map for the study área (modified, IGME).

desestimada tras llevar a cabo, recientemente, una intervención arqueológica sobre el mismo y comprobar que no existen depósitos de terraza en el emplazamiento de El Barandiallu (Álvarez-Alonso, 2013). Hay que matizar, no obstante, que la ladera en la que se localiza el yacimiento está afectada por fenómenos de solifluxión, presentando morfologías de glacis, pero no un modelado fluvial. Así, el yacimiento se localiza dentro de un horizonte edáfico desarrollado a expensas de un paquete de arcillas rojas triásicas, que presentan evidencias de hidromorfismo.

El descubrimiento se produjo en 1988 cuando el arqueólogo Rogelio Estrada localizó un numeroso conjunto lítico durante las obras del gaseoducto Burgos-Cantabria-Asturias. Este conjunto fue aumentando durante sucesivas visitas al lugar entre 1988 y 1989, coincidiendo con las labores de arado, hasta llegar a reunir una colección superior a las 1000 piezas, para las que se barajó su adscripción al Musteriense de Tradición Achelense A (Estrada y Jordá, 2004). Con posterioridad, en julio de 2012, llevamos a cabo una intervención arqueológica sobre una superficie total de $12 \mathrm{~m}^{2}$, en la que se hallaron 348 piezas más ${ }^{1}$ (Álvarez-Alonso 2013).

\section{3. - LA EXCAVACIÓN}

En total se excavaron cuatro sondeos, dos de $2 \times 2$ $\mathrm{m}$ y otros dos de $1 \times 2 \mathrm{~m}$, alcanzando una superficie de $12 \mathrm{~m}^{2}$. Los sondeos A y C, localizados a igual cota (128 m.s.n.m.), mostraron mayor densidad de material, mientras que el sondeo B -a una cota inferior- mostró menos densidad, y el D -a una cota superior-, prácticamente no presentaba hallazgos, aportando una estratigrafía ligeramente diferente a los anteriores (Álvarez-Alonso,
2013) (Fig.3). La excavación se realizó mediante el levantamiento de capas artificiales de espesor inferior a $5 \mathrm{~cm}$, adaptándose siempre a las variaciones de sedimento y estratos identificados. Cada sondeo se dividió en cuadros de $1 \mathrm{~m}^{2}$ subdivididos en nueve sectores, para llevar un mayor control de los hallazgos. Todo el sedimento obtenido fue cribado con una malla de $2 \mathrm{~mm}$ de luz. Esta metodología permitió contrastar los datos de las recogidas en superficie, además de comprobar la dispersión del material y la densidad e integridad del yacimiento (Álvarez-Alonso, 2013).

Tras los trabajos, quedó establecida una secuencia estratigráfica compuesta por un nivel superficial edáfico de unos 25-40 cm de espesor variable según la zona, donde se localiza la totalidad del material, excepto alguna pieza que se halló en las arcillas triásicas, fruto de la existencia de grietas de desecación donde se ha filtrado material del nivel superior. Este horizonte edáfico, alterado por la acción del arado -nivel 1- se ha desarrollado a partir de un paquete de arcillas estériles de origen triásico -nivel 2- (Fig.4-5) (Álvarez-Alonso, 2013).

\section{4. - EL CONJUNTO LÍTICO DE EL BARANDIALLU}

Esta colección está formada por 1426 piezas -1090 recogidas en superficie y 336 en excavación-. El área de dispersión máxima en superficie lo hemos establecido en unos $9000 \mathrm{~m}^{2}$ aunque la mayor concentración se localiza en la zona excavada², un área no superior a los $3000 \mathrm{~m}^{2}$. Este conjunto se puede analizar unitariamente, independientemente de su procedencia (superficie o excavación) ya que, además de tener su origen en un único horizonte estratigráfico, no presenta diferencias tecnotipológicas a nivel interno que nos permitan pensar en la existencia de agregados líticos 

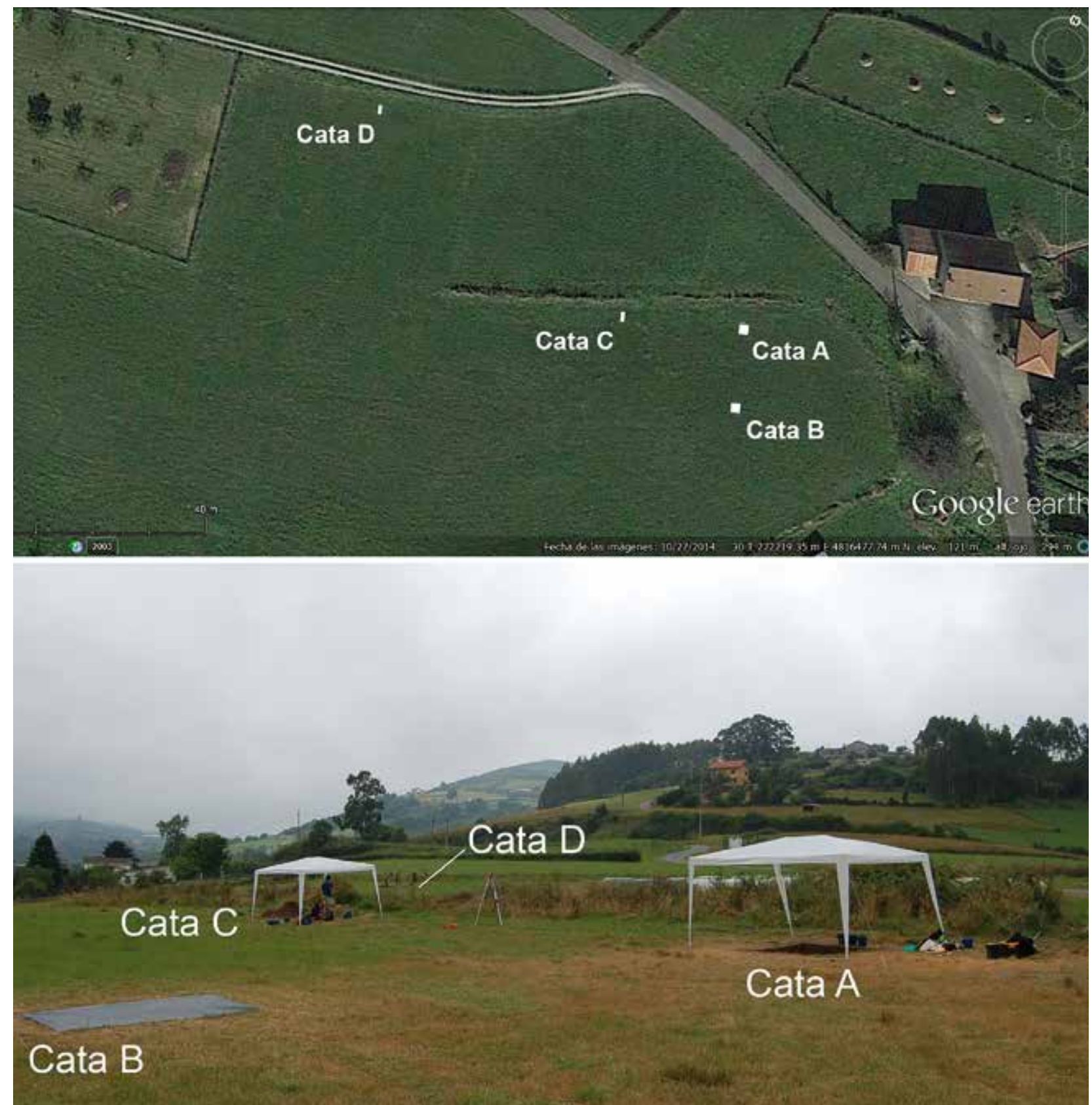

Fig. 3. Ortofoto con la localización de los sondeos (arriba). Imagen de la excavación con las distintas zonas (abajo). / Orthophoto with the location of the sondages (above). Image of the excavation with the different areas (below).

de distintas cronologías o fases. Como hemos explicado con anterioridad, el horizonte arqueológico está desmantelado por la acción del arado, y la posición del material, en superficie o dentro del nivel edáfico, resulta aleatoria y derivada de la actividad agrícola ya que, dicho horizonte -con presencia también de materiales medievales y modernos- se asienta directamente sobre un depósito triásico a expensas del cual se ha formado (Álvarez-Alonso, 2013).

Individualizar la procedencia de los materiales nos permite, en algunos casos, ver las diferencias existentes, así como el sesgo en el muestreo superficial (porcentajes de materia prima o selección por tamaños) (Fig.6), siendo de utilidad en el análisis tafonómico y en la interpretación del grado de integridad de la muestra, de cara a un posterior estudio espacial. Así, mientras más del 50\% del material recogido en superficie tiene unas dimensiones $(L)$ superiores a $42 \mathrm{~cm}$, el $50 \%$ de la muestra de excavación se sitúa por debajo de $31 \mathrm{~cm}(\mathrm{~L})$. En este conjunto hay una gran variedad de elementos pertenecientes a las distintas fases de varias cadenas operativas, además de encontrarnos con hasta 12 percutores. 


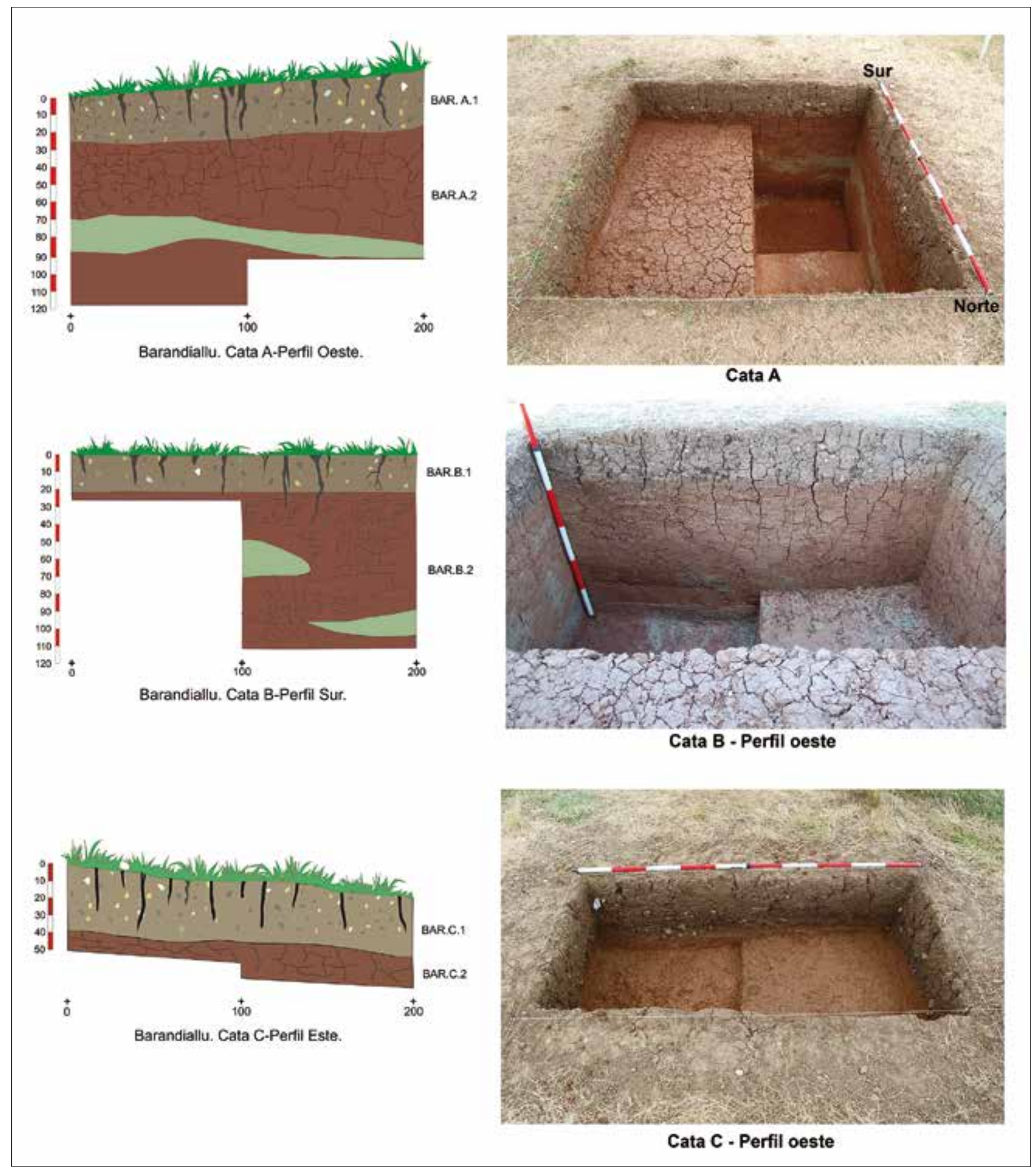

Fig. 4. Estratigrafía e imágenes de las zonas $A, B$ y C, una vez concluida la excavación. / Stratigraphy and images of the $A, B$ and $C$ zones, once the excavation was finished. (Álvarez-Alonso, 2013) 
LAS CADENAS OPERATIVAS LÍTICAS DE EL BARANDIALLU (ASTURIAS, ESPAÑA):

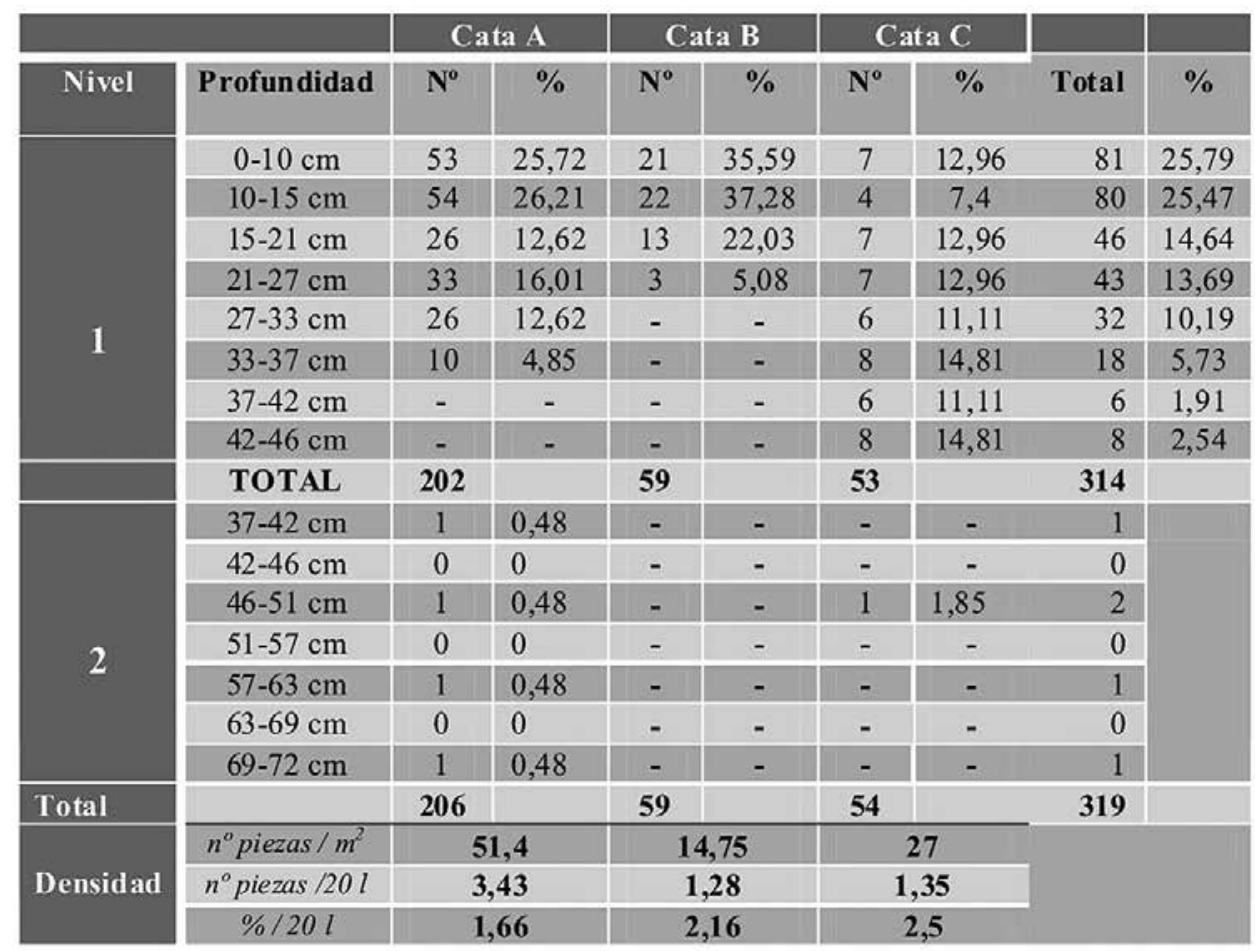

Fig. 5. Tabla de distribución y densidad de la industria lítica (Álvarez-Alonso, 2013). / Table showing the distribution and density of the lithic industries (Álvarez-Alonso, 2013).

\begin{tabular}{l|c|c|c|c|} 
& L mín. & L máx. & Media & Mediana \\
\hline Cuarcita superficie & 16,68 & 149,06 & 57,53 & 54,31 \\
\cline { 2 - 5 } Cuarcita excavación & 14,95 & 79,36 & 33,72 & 30,9 \\
\cline { 2 - 5 } Sílex superficie & 35,97 & 62,01 & 47,33 & 42,73 \\
Sílex excavación & 8,67 & 52,32 & 21,64 & 21,62 \\
\hline
\end{tabular}

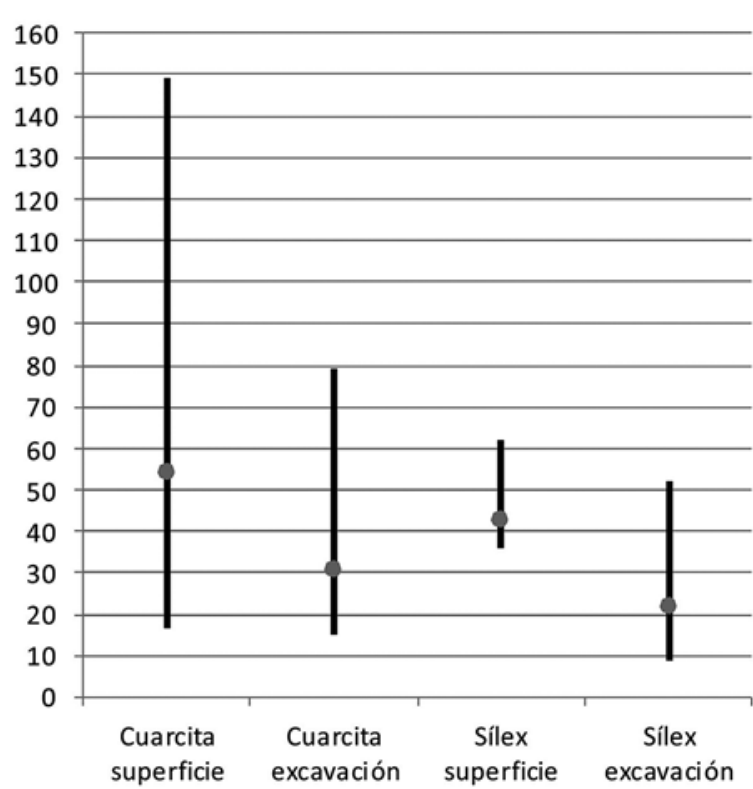

- Mediana

Fig. 6. Tabla con la representación de todos los elementos en función de su eje mayor o (L), en la que se aprecia la diferencia entre el tamaño de los materiales localizado en superficie y en la excavación., así como entre la cuarcita y el sílex. / Table showing the representation of all elements in relation to its $L$ axis, in which it is possible to appreciate the size difference between the surface findings and the lithics found in the excavation, as well as between quartzites and flint. 


\subsection{Materias primas}

La cuarcita es el material más abundante $(94,4 \%)$, seguida del sílex (3,71\%) y el cuarzo (1,82\%) (Fig.7), y únicamente está presente en formato «canto rodado» procedente del entorno inmediato al yacimiento, donde se localizan los dos principales focos de este material en la zona: los conglomerados de origen jurásico en la parte septentrional del valle, y los depósitos de origen triásico de la parte más meridional, también constituyendo conglomerados. Estos últimos, pertenecientes al Buntsandstein (Triásico inferior), afloran en la ladera situada frente a El Barandiallu y poseen numerosos cantos de cuarcita que, al igual que en el primer caso, han sido progresivamente desmantelados por la erosión y la incisión fluvial cuaternaria. Esta circunstancia ha provocado la incorporación de numerosos cantos rodados al lecho fluvial, pudiendo también encontrarse en algunos depósitos colgados a una cota superior al cauce actual. Por su parte, los conglomerados cuarcíticos jurásicos del alto del Castiello, aportan material a los coluviones localizados en ladera, en la margen izquierda del valle, formando sucesivos resaltes y glacis que recubren parcialmente el Triásico. Todas estas cuarcitas son accesibles en un radio de $1 \mathrm{~km}$ desde el yacimiento, caracterizándose por ser cantos muy bien redondeados de tonos grisáceos-verdosos y rojizos (Fig.8). En el enclave de El Barandiallu no hemos localizado depósitos coluvionares o aluviales con estos materiales. En consecuencia, podemos afirmar que la totalidad del material cuarcítico presente en el yacimiento fue aportado antrópicamente desde un entorno inmediato, donde sí se localizan estos depósitos. Este caso parece equiparable al de El Habario, donde se explotan conglomerados cuarcíticos (Carrión y Baena, 2005), pareciendo así una estrategia repetida por los grupos neandertales cantábricos.

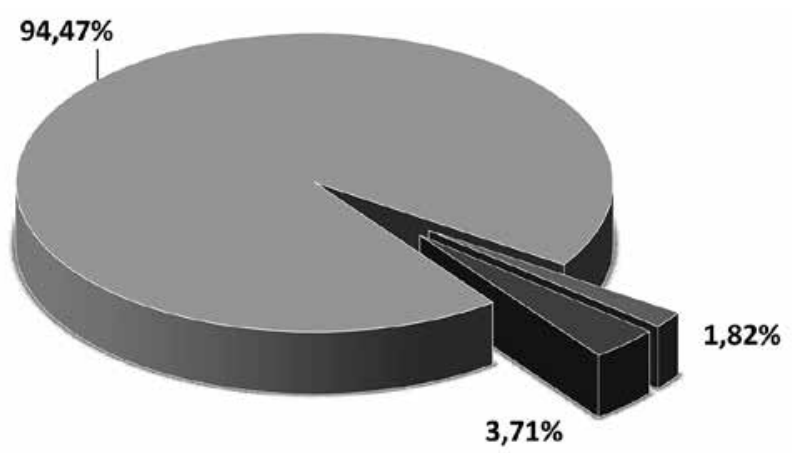

n sílex = cuarcita cuarzo

Fig. 7. Porcentajes de materias primas. / Raw materials percentage

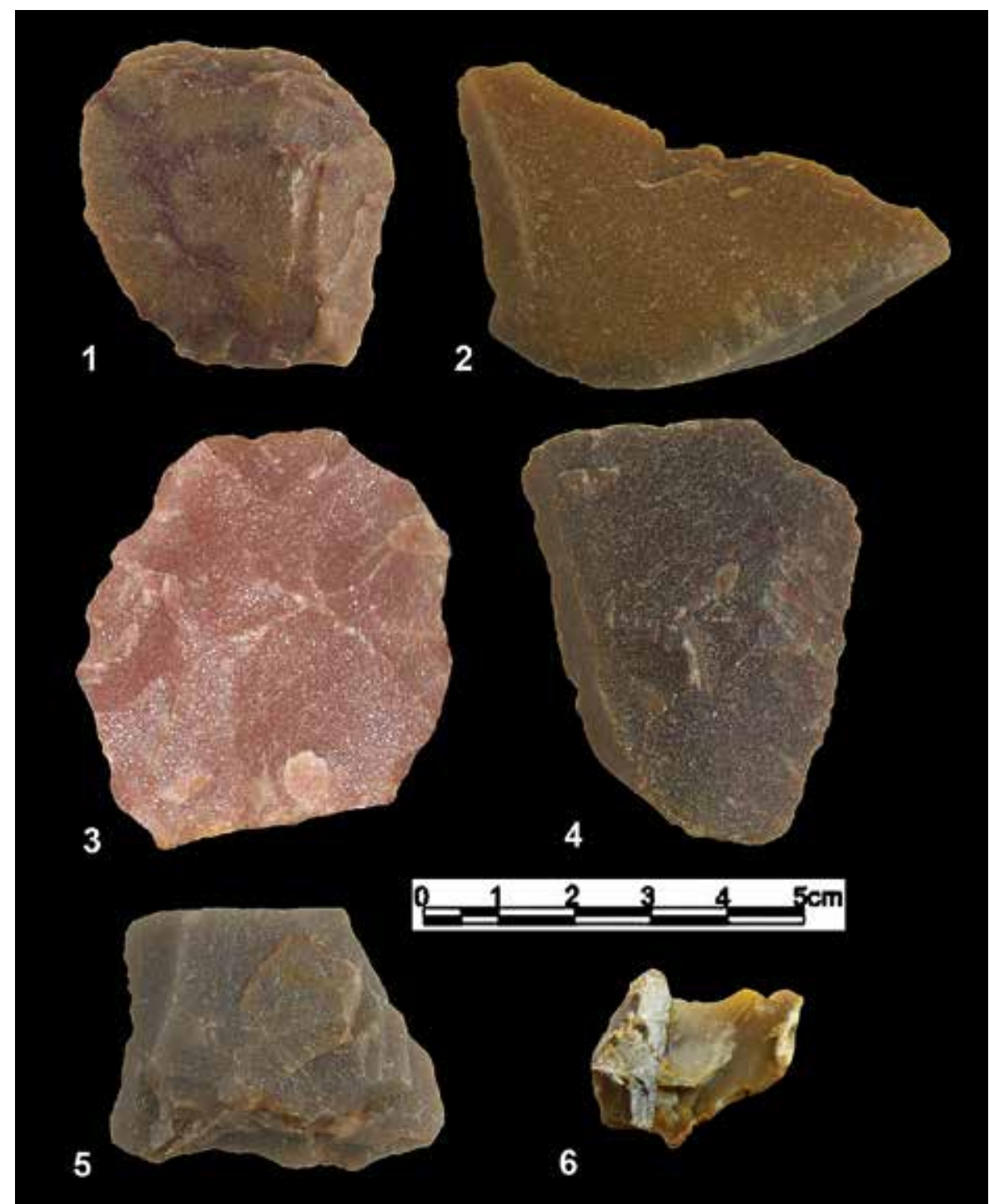

Fig. 8. Distintos tipos de cuarcita (1-5) y sílex de Piloña (6) presentes en el conjunto. / Different types of quartzite $(1-5)$ and Piloña Flint (6) documented in the assemblage. 
En el conjunto también destaca la presencia de distintas variedades de sílex, cuya procedencia se localiza a varios kilómetros. En total hay 53 piezas de sílex: 23 identificadas como «Piedramuelle»; 9 como «Piloña» (Fig.8) y 21 indeterminadas debido a su grado de alteración (Álvarez-Alonso, 2013; Tarriño et al., 2013; Santamaría et al., 2011). La identificación de la fuente de aprovisionamiento resulta de gran interés, ya que podemos trazar rutas concretas y poner en relación El Barandiallu con yacimientos musterienses cercanos a estos dos afloramientos, como son La Viña o El Sidrón. El hecho de que toda la materia prima sea aportada desde un entorno inmediato (cuarcita), o más lejano (sílex), hace que El Barandiallu pueda encajar con una ubicación o emplazamiento estratégico seleccionado por su

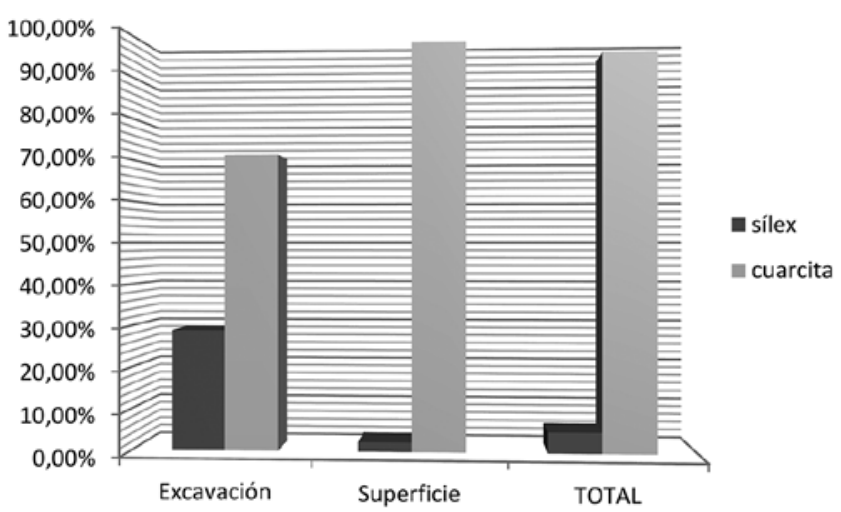

Fig. 9. Distribución de las materias primas en función de su localización en superficie o en la excavación. / Distribution of the raw materials in relation to its location in surface or in the excavation. posición dentro de un territorio definido. Por otra parte, esta circunstancia también confirma que se trata de un emplazamiento primario y no de un yacimiento derivado.

Hay que destacar que, entre la muestra procedente de la excavación, el porcentaje de sílex es mayor que en superficie (Fig.9), debido principalmente a que el tamaño de las piezas de sílex es mucho menor que la media del utillaje y del conjunto de piezas de cuarcita (Fig.6). Esto es síntoma del sesgo que imprimen las recogidas de material lítico en superficie a la caracterización de los yacimientos, marcadas por una fuerte selección del material más llamativo o de mayor tamaño.

\subsection{Cadenas operativas de débitage}

En este grupo englobamos las cadenas operativas destinadas a la producción de lascas, empleándose diferentes métodos de talla, que han generado restos de distinto tipo: lascas simples, núcleos, productos de acondicionamiento y avivado.

Bajo este epígrafe agrupamos la mayor parte del conjunto. Así, excluyendo el macroutillaje $(n=26)$ del total de 1426 piezas, pero manteniendo todos los elementos retocados sobre lasca, vemos que, de las 1400 piezas restantes, el 68\% son lascas, el 1,4\% lascas Levallois, el $12,2 \%(n=171)$ son núcleos y el $5,7 \%$ productos de avivado y acondicionamiento (principalmente flancos y aristas de cornisa); a los que sumamos 12 percutores.

A su vez, bajo el concepto de débitage distinguimos, por una parte, los productos de talla sean del tipo que sean y por otra los núcleos (Fig.10).

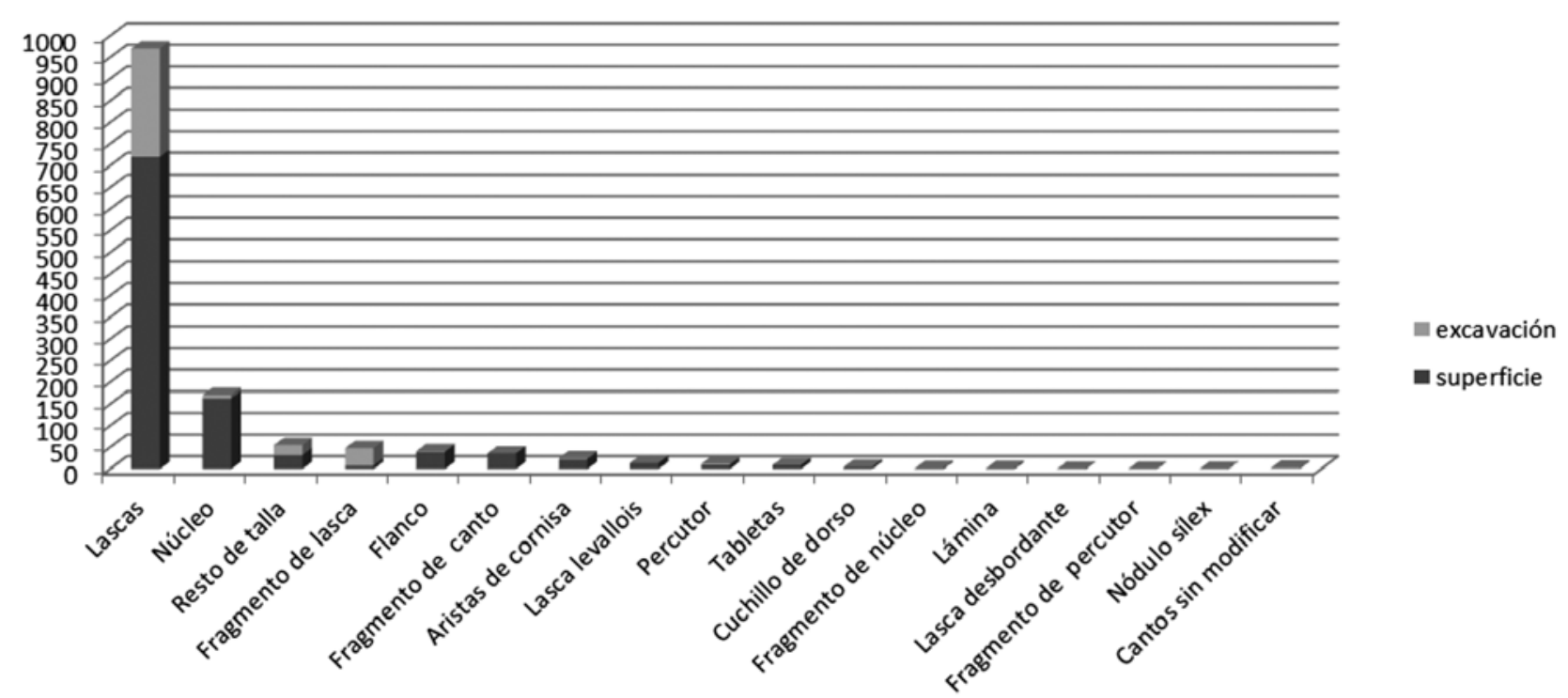

Fig. 10. Productos de talla, núcleos y percutores. / Knapping products, cores and hammers. 


\subsubsection{Los productos de talla}

Fundamentalmente, la información tecnológica que obtenemos de los productos de talla proviene del análisis de sus caras dorsales (corticalidad y tipología, orientación y número de las extracciones identificadas), de sus plataformas de percusión (talones y ángulos de percusión), y finalmente de su clasificación e inclusión dentro de cada una de las fases de la cadena operativa: lascas simples, productos de avivado, productos de acondicionamiento, desechos de talla, debrís... (Fig. 11). El análisis de estos atributos, junto con el estudio de los núcleos, nos puede ayudar a completar la información tecnológica sobre los métodos de talla empleados, el grado de aprovechamiento de la materia prima y el tipo de gestión que de esta se hace, mediante la implementación de distintas estrategias y técnicas de talla.

En primer lugar, el grado de corticalidad ha sido medido sobre 1103 soportes (incluyendo lascas simples, retocadas, productos de acondicionamiento, etc), de las cuales el 53,59\% no tienen córtex, el 3,8\% son de decorticado primario (lascas de desbastado) y el $42,79 \%$ de decorticado secundario. De entre las lascas de decorticado secundario $(n=472)$, el $83,89 \%$ tienen menos de un 50\% de córtex en su cara dorsal (Fig.12). Los restos corticales presentes en las caras dorsales analizadas se localizan mayoritariamente en el tercio inferior de la pieza (zonas proximal o lateral), siendo aproximadamente tan solo un $15 \%$ de ocasiones cuando el córtex se presenta en la mitad distal de la pieza.

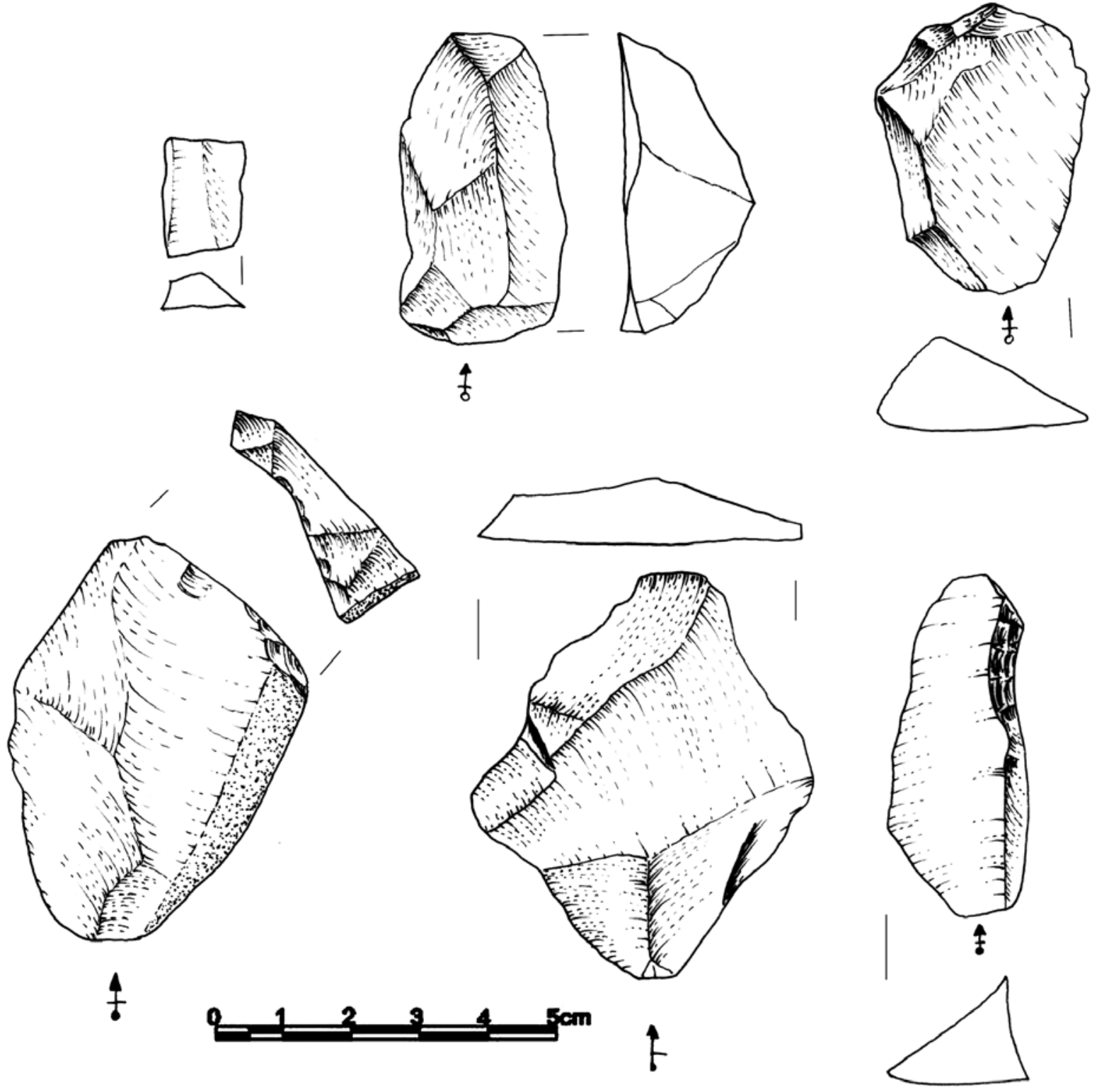

Fig. 11. Distintos productos de acondicionamiento (aristas de cornisa, flancos y tabletas) y la única lámina presente en el conjunto. / Different conditioning products (cornice edges, flanks and tablets) and the single blade present in the assemblage. 


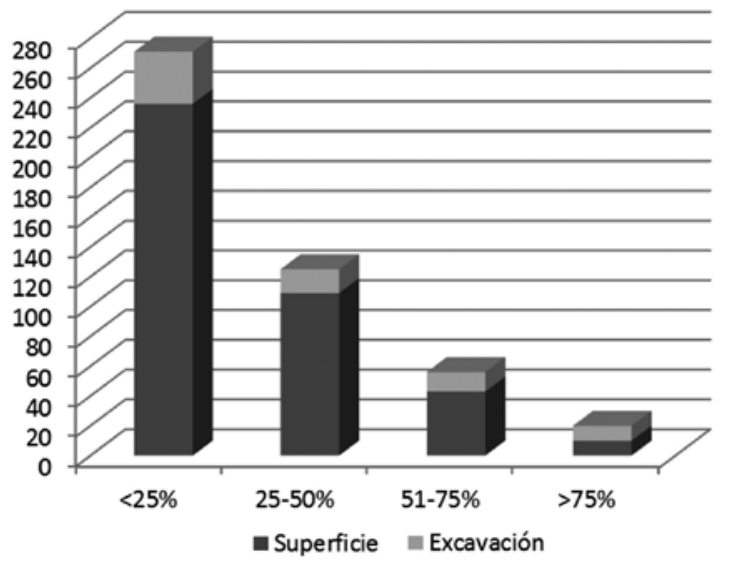

Fig. 12. Representación del grado de corticalidad en los productos de decorticado secundario. / Representation of the corticality degree in secondary decorticating products.

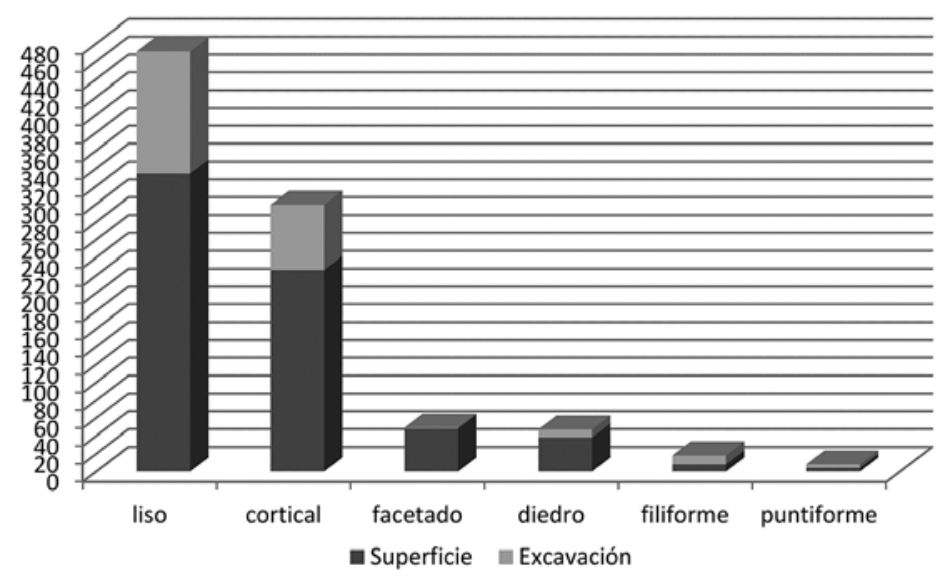

Fig. 13. Representación en de los tipos de talón documentados. / Representation of the types of the documented hells.

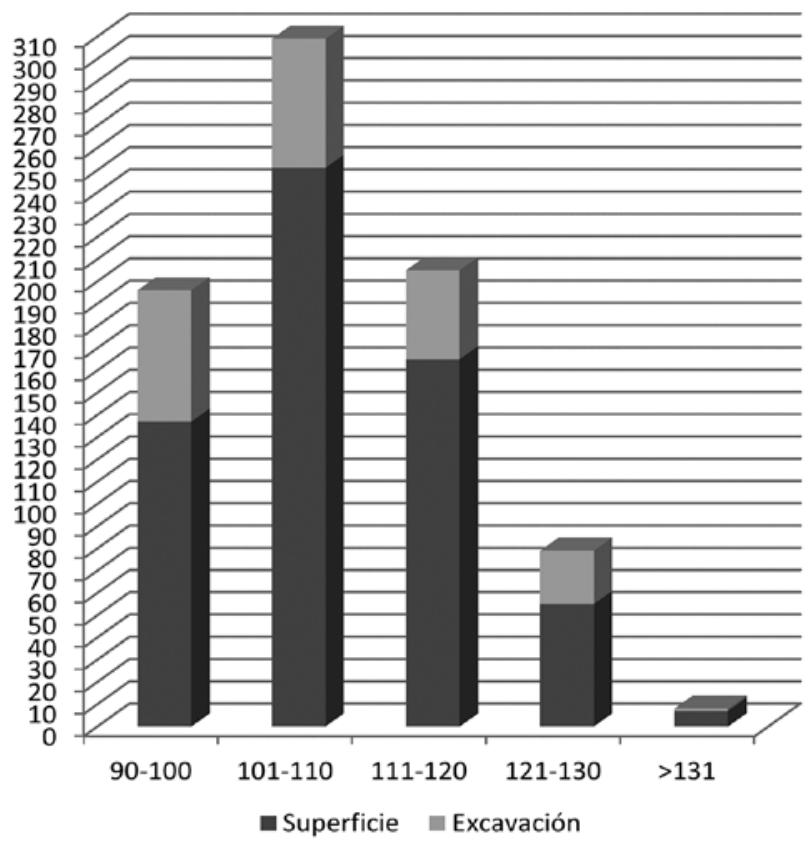

También se ha analizado el número, disposición y organización de las extracciones, para tratar de identificar los patrones y métodos de talla. Así, se ha identificado el tipo de negativos en 494 productos, fundamentalmente lascas, resultando que un 47,57\% son centrípetos; el 31,78\% unipolares convergentes; el $10,72 \%$ unipolares paralelos; el 7,60\% perpendiculares y el $2,22 \%$ bipolares, apreciándose la importancia de los métodos unipolares y centrípetos.

En cuanto a las plataformas de percusión, el número de talones reconocibles asciende a 892, siendo mayoritarios los lisos (52,8\%), seguidos de los corticales (33,52\%) (Fig.13). El índice de facetado (If) es del $10,76 \%$, mientras que el índice de facetado estricto (Ifs) se sitúa en el 5,49\%. En cuanto a los ángulos de percusión (formado por el talón y la cara ventral) se han podido identificar en un total de 797 lascas. El porcentaje mayor se sitúa en los valores comprendidos entre $101-110^{\circ}(38,77 \%)$; seguidos de los comprendidos entre $121-130^{\circ}(25,72 \%)$ y a continuación los situados entre $90-100^{\circ}$ (24,59\%) (Fig.14).
Fig. 14. Representación en de los tipos de ángulos de talla documentados. / Representation of the documented knapping angles. 
Por otra parte, la modularidad de las lascas, establecida a partir de 210 soportes enteros, nos ofrece una longitud media de $29 \mathrm{~mm}$, con un máximo de $72 \mathrm{~mm}$ y un mínimo de 11, siendo la mediana 27 mm (Fig. 15).

A priori, estos datos muestran unos productos con escasos indicios de preparación de planos de percusión, utilizando preferentemente plataformas planas o lisas con una alta representación de superficies de percusión corticales.

Por otra parte, las superficies dorsales de los productos de talla muestran que, a pesar de existir un número elevado de piezas con restos corticales, se trata de porcentajes bajos situados preferentemente en las zonas proximales y laterales, pudiendo corresponder con una talla preferentemente centrípeta o unipolar. Si

\subsubsection{Los núcleos}

Se documentan 171 núcleos, destacando un número elevado realizado sobre lascas corticales: grandes lascas y segmentos de canto. En primer lugar, hemos clasificado los núcleos atendiendo a su esquema operativo básico y sus superficies de extracción (unifaciales, bifaciales, multifaciales), para posteriormente clasificarlos en función del método de talla desarrollado a partir de cada uno de los esquemas anteriores (discoide, Levallois, NUPC...). El objetivo es establecer una primera categoría más genérica, adecuada a la morfología de las matrices (cantos rodados y lascas), que permita categorizar y observar de una manera más precisa cómo se lleva a cabo la gestión de todas estas morfologías. La mayoría de los núcleos de El Barandiallu han resultado

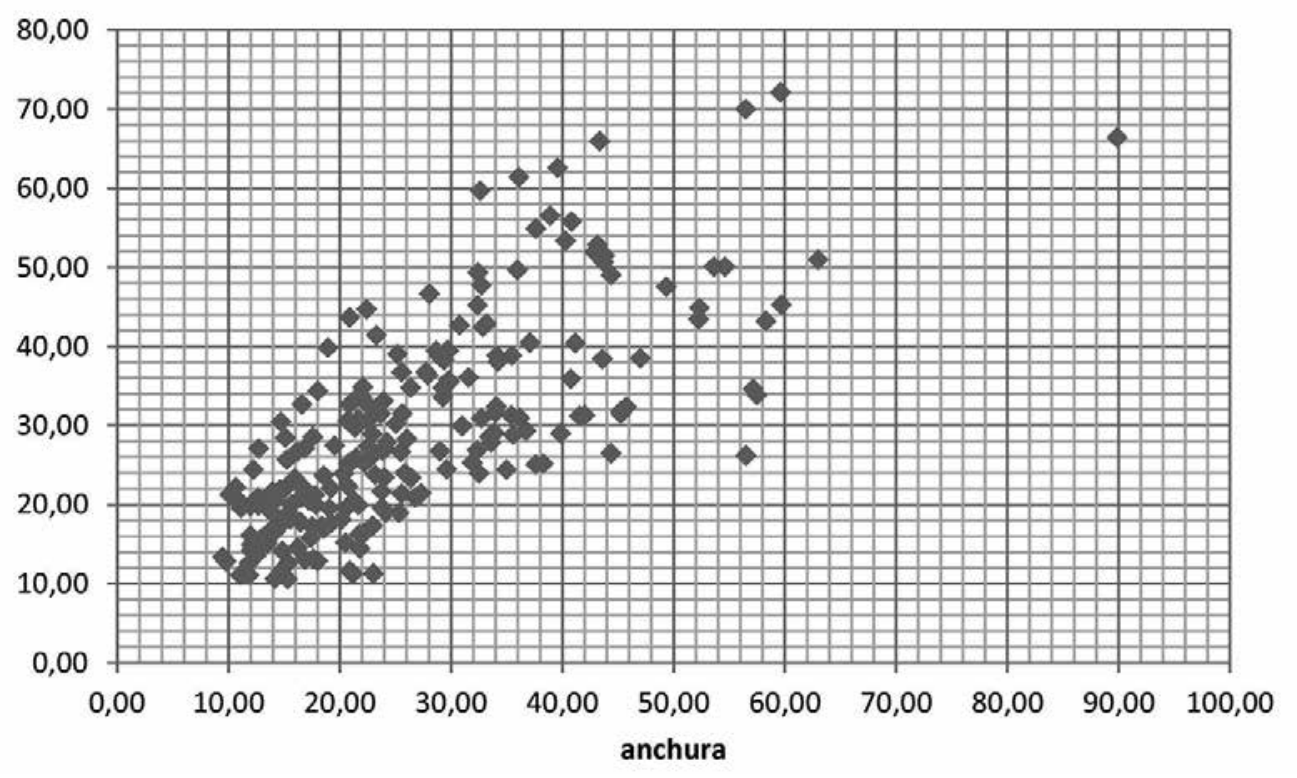

Fig. 15. Grafica de tamaños (L/a) sobre lascas completas. / Grahp showing L/W axes using complete flakes. bien los elementos centrípetos son mayoritarios, destaca el elevado número de piezas con superficies dorsales unipolares.

Además, no descartamos que una fase previa de la preparación de los núcleos, que implique su reducción de tamaño, desbastado inicial y preparación de los cantos, haya sido realizada en el lugar de captación, ya que esta fase no está bien documentada en el conjunto. Esto tendría lógica, tratándose de una materia prima que debe ser transportada hasta el emplazamiento seleccionado, por lo que cualquier reducción de volumen, y de peso, economizaría el esfuerzo del transporte.

En resumen, los productos nos indican una variedad relativamente amplia de etapas dentro de los procesos talla que además parecen adecuarse a un tipo de gestión de la materia prima muy concreta. ser de tipo unifacial, seguidos de los bifaciales, con una presencia moderada de núcleos multifaciales.

\subsection{2.a Núcleos unifaciales}

Suelen realizarse sobre la cara ventral de una lasca, reservando la cara cortical como superficie de percusión. La mayoría de estos núcleos son de tipo discoide unifacial, pero en algunos de ellos se percibe una somera preparación de la cara cortical, que los aproximaría morfológicamente a los núcleos Levallois (Boëda, 1993, 2006; Bourguignon et al. 2006), aunque más bien encajan en la definición del tipo "discoides unifaciales jerarquizados" (Terradas, 2003). En este grupo también se incluyen los núcleos de tipo NUPC -núcleos unidireccionales sobre plataforma cortical- (Arias, 1991). 


\subsection{2.b Núcleos bifaciales}

En su mayoría han sido realizados sobre lascas, aunque también hay algún ejemplo de utilización de fragmento de canto rodado. En todos estos casos, la bifacialidad parece implicar un grado mayor de aprovechamiento ya que los productos obtenidos, tanto en tamaño como en morfología, son muy similares en los núcleos unifaciales y bifaciales. Por esta razón, los núcleos bifaciales no parecen ser una estrategia técnica distinta a los unifaciales, sino más bien una intensificación en la explotación con respecto a los anteriores, en la que el condicionamiento de la matriz, también juega un papel importante. Los métodos de talla desarrollados a partir de esquemas bifaciales, son fundamentalmente de tipo discoide y Levallois, al igual que en el caso anterior. De este modo, creemos que ambos tipos, unifaciales y bifaciales, están muy ligados entre sí con la excepción del tipo NUPC.

\subsection{2.c Núcleos multifaciales}

Están realizados preferentemente a partir de cantos o fragmentos de canto con varias facetas, y formas poliédricas (de tetraedro a hexaedro), lo cual condiciona la morfología resultante y el tipo de explotación empleada. Dentro de este grupo los métodos de talla identificados son principalmente de tipo SSDA y Quina, donde la recurrencia en la explotación, la alternancia de roles entre superficies de lascado y percusión, o su jerarquización, no siempre está muy bien definida. Por esta razón, creemos más apropiado referirnos a ellos genéricamente como núcleos multifaciales, no entrando a un grado de definición mayor, ya que se trata del tipo menos representado en el registro lítico de El Barandiallu. Algunos de estos núcleos encajarían en otras definiciones que se han dado para el ámbito pirenaico, como núcleos prismáticos (Thiébaut et al., 2012)

En resumen, podemos indicar que los esquemas de tipo unifacial y bifacial representan casi el $75 \%$ de los núcleos de El Barandiallu, siendo fundamentalmente discoides, Levallois y NUPC.

\subsubsection{Los métodos de talla}

A la hora de analizar los métodos de talla implementados en El Barandiallu, vemos que en el conjunto de núcleos hay mayoría de tipo discoide, que suponen el $46 \%$ del total, mientras que también hay un número importante de núcleos sobre superficie de debitage alterno -SSDA- (16\%) y Levallois (12\%), con bastantes núcleos extremadamente agotados y, por tanto, indeterminados. Entre los discoides, al menos 16 son unifaciales sobre plataforma cortical, realizados directamente sobre canto (una mitad de canto rodado). Se trata de un tipo muy específico: medio canto partido por la mitad y explotado periféricamente sobre la superficie de lascado. Este método ha sido observado también en
Bañugues y se aproxima al tipo NUPC (Álvarez-Alonso et al., 2014), siendo una variedad de explotación a partir de cantos de cuarcita de tipo globular, muy abundantes en los conglomerados triásicos y jurásicos del entorno de El Barandiallu.

Así, en lo que a las matrices se refiere, hemos observado una utilización sistemática de cantos, fragmentos de canto y lascas corticales procedentes de cantos generalmente globulares que han sido convertidos en núcleos. Esta preferencia es síntoma de la abundancia de este tipo de formatos entre la cuarcita local, a la cual se adaptan los métodos de talla. Como resultado, podemos observar una gradación en el uso de soportes, que van desde el canto globular hasta lascas de tamaño mediano. En todos los casos se ha desarrollado un planteamiento conceptual similar: golpear sobre la cara cortical, sin preparación o ligeramente preparada, resultando núcleos que podemos clasificar como NUPC, Levallois o discoides en distintos tipos, en función del soporte de partida y su grado de aprovechamiento, debido a la intensidad o recurrencia de las series de talla. Este tipo de explotación, aprovechando las caras corticales de cantos y lascas, y utilizando ángulos naturales cercanos a $90^{\circ}$ en el primer caso, se ha registrado en más yacimientos musterienses cantábricos, como Las Monedas o El Habario (Carrión y Baena, 1998, 2005). En la cueva de La Flecha, también se documenta una utilización sistemática de cantos rodados, pudiendo estar relacionado con una captación y acceso inmediato a la fuente materia prima -conglomerados cuarcíticos-, resultando un tipo de núcleos y productos adaptados y derivados de esta circunstancia (Castanedo, 2001).

Los métodos de talla empleados en El Barandiallu y la morfología resultante de los núcleos son consecuencia directa de una estrategia donde abundan los cantos rodados que han sido partidos por la mitad o fracturados en distintas partes, así como las grandes y pequeñas lascas corticales resultado de su explotación.

\subsection{Cadenas operativas de façonage o confi- guración}

El $32,53 \%(n=464)$ del conjunto son piezas retocadas $(n=438)$ o macroutillaje $(n=26)$. Para realizar su estudio las dividimos en estos dos grupos: utillaje retocado sobre lasca y macroutillaje (bifaces, hendedores y cantos trabajados).

\subsubsection{Utillaje sobre lasca}

Destacan por encima del resto, el grupo de las raederas (raederas -26,95\%- + lascas retocadas -15,75\%-) con un $42,7 \%$, predominando las simples sobre las dobles. El siguiente grupo en importancia son las piezas denticuladas -39,26\%-. Todas estas piezas han sido elaboradas en cuarcita (Figs.16-17). 
Morfotipos

\begin{tabular}{|c|c|c|c|}
\hline \multicolumn{4}{|l|}{ Raederas } \\
\hline & Lateral & 79 & $18,03 \%$ \\
\hline & Doble & 6 & $1,36 \%$ \\
\hline & Convergente & 6 & $1,36 \%$ \\
\hline & Transversal & 26 & $5,93 \%$ \\
\hline Lascas retocadas & & 69 & $15,75 \%$ \\
\hline Escotaduras & & 100 & $22,83 \%$ \\
\hline Denticulados & & 72 & $16,43 \%$ \\
\hline Lascas truncadas & & 11 & $2,51 \%$ \\
\hline \multicolumn{4}{|l|}{ Útiles compuestos } \\
\hline & Raedera + escotadura & 8 & $1,82 \%$ \\
\hline & Raedera + denticulado & 3 & $0,68 \%$ \\
\hline & Raedera + rabot & 1 & $0,22 \%$ \\
\hline & Denticulado + truncadura & 1 & $0,22 \%$ \\
\hline & Lasca truncada + escotadura & 1 & $0,22 \%$ \\
\hline & Raspador + ecaillé & 1 & $0,22 \%$ \\
\hline Diversos & & 11 & $2,51 \%$ \\
\hline Rabot & & 1 & $0,22 \%$ \\
\hline \multicolumn{4}{|l|}{ Puntas } \\
\hline & Musteriense & 2 & $0,45 \%$ \\
\hline & Tayac & 1 & $0,22 \%$ \\
\hline Piezas astilladas & & 8 & $1,82 \%$ \\
\hline Raspadores & & 11 & $2,51 \%$ \\
\hline Buriles & & 3 & $0,68 \%$ \\
\hline Perforadores & & 9 & $2,05 \%$ \\
\hline Raclette & & 1 & $0,22 \%$ \\
\hline Bec & & 6 & $1,36 \%$ \\
\hline Tranchet & & 1 & $0,22 \%$ \\
\hline
\end{tabular}

Fig. 16. Lista de los útiles retocados sobre lasca. / List of the retouched pieces made on flakes.
Destacamos que en el $81,96 \%$ del utillaje predominan los retoques de tipo denticulado y simple (raederas y denticulados), siendo el resto del utillaje de mucha menor importancia en el conjunto total. A pesar del elevado número de piezas presentes, solo se contabilizan dos puntas musterienses típicas, que ayudan a completar la atribución crono-cultural desde un punto de vista tipológico. Por otra parte, destaca la notable representación del llamado «grupo tipológico del Paleolítico superior» con un 3,19\% (raspadores y buriles), seguidos de otros tipos menos habituales en el Musteriense como los perforadores o raclettes, que aparecen en un porcentaje aún más bajo. Estos útiles, lejos de considerarse intrusiones, son elementos que, aunque escasos, no son ajenos al Paleolítico medio, como puede observarse en otros conjuntos de factura similar asignados al MTA (Soressi, 2002). En El Barandiallu no se han elaborado sobre soportes laminares, prácticamente ausentes del conjunto, aunque preferentemente se ha seleccionado el sílex para su elaboración. Es por ello que, podemos plantear la existencia de cadenas operativas diferentes, encaminadas a la elaboración de un utillaje más genérico en materias primas locales (cuarcita) y de elementos más concretos y escasos en materias exógenas (sílex). En consonancia, con esta interpretación el utillaje de sílex parece haber sido aportado al yacimiento, ya que apenas se han registrado restos de talla en esta materia prima, y todo apunta a que se trata de productos finales o que se introducen en un estado avanzado de elaboración dentro de sus respectivas cadenas operativas.

En lo que se refiere al utillaje retocado sobre lasca, se trata de un tipo y proporción de útiles de indiscutible filiación musteriense (Fig.18). 


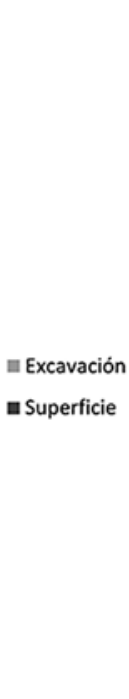

Fig. 17. Gráfica con los útiles sobre lasca en función de su localización. / Graph showing the retouched pieces made on flakes in relation to their location.
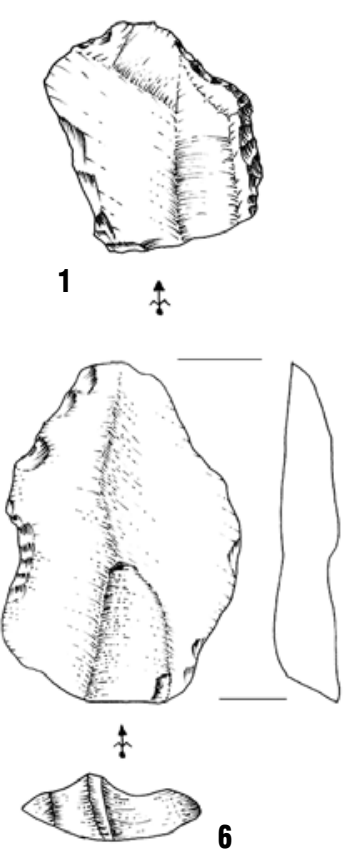
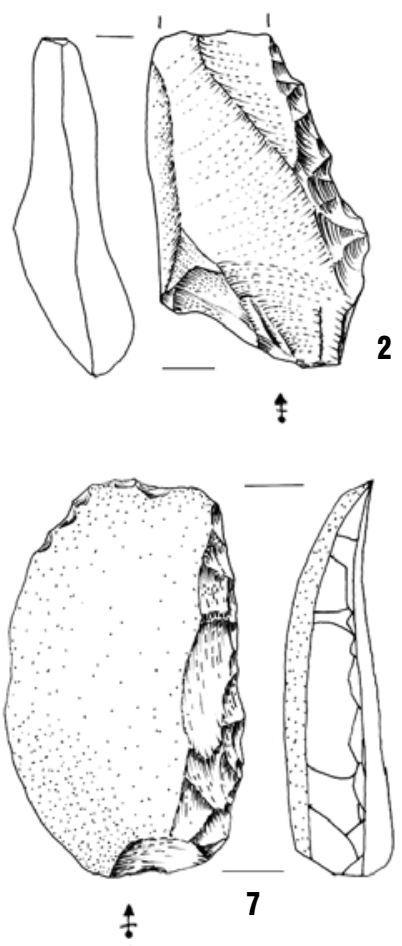
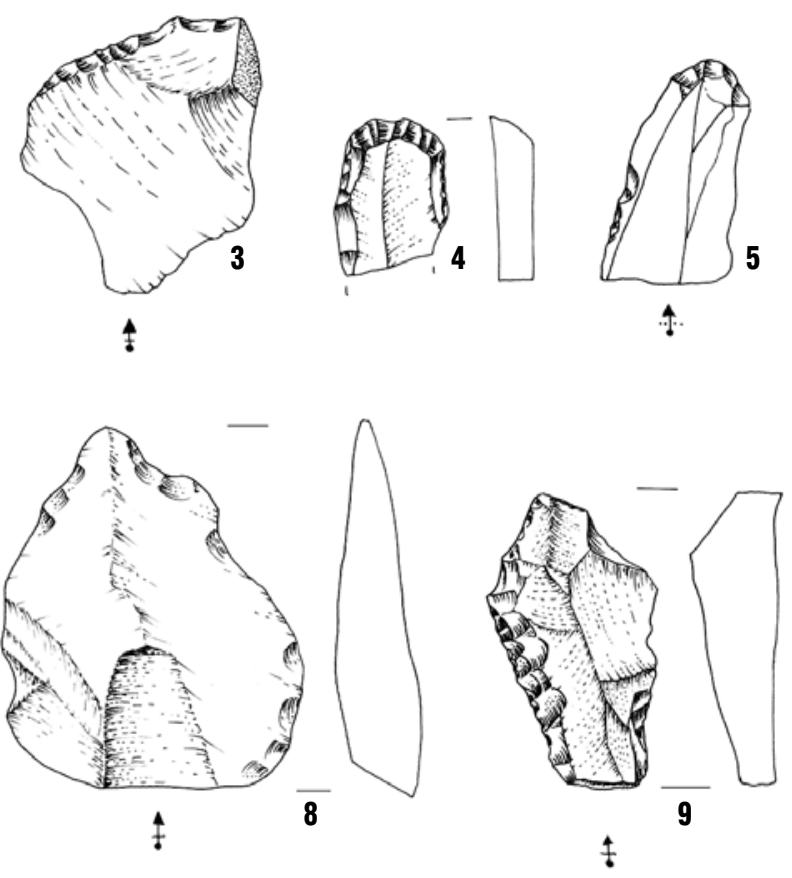

Fig. 18. Soportes retocados: raederas (1-3,7,9), denticulados (1-3,7,9), raspadores (4-5). / Retouched basis: sidescrapers (1-3,7,9), denticulates (1-3,7,9), endscrapers (4-5).

\subsubsection{Macroutillaje}

Todos los elementos de macroutillaje han sido realizados en cuarcita: 9 hendedores, 5 bifaces, 7 cantos trabajados, 3 masivos con extracciones y 2 picos triedros, destacando un mayor número de hendedores que de bifaces, aunque tampoco es una diferencia muy significati- va. Mientras en el primer estudio publicado de una parte del material de El Barandiallu (Estrada y Jordá, 2004) se mencionan 50 piezas de macroutillaje (21 bifaces, 12 hendedores, 11 triedros y 6 cantos trabajados) (Fig.19), tras nuestro análisis, esa cifra se ha visto notablemente reducida, seguramente por la reinterpretación de alguna de estas piezas como núcleos bifaciales. 


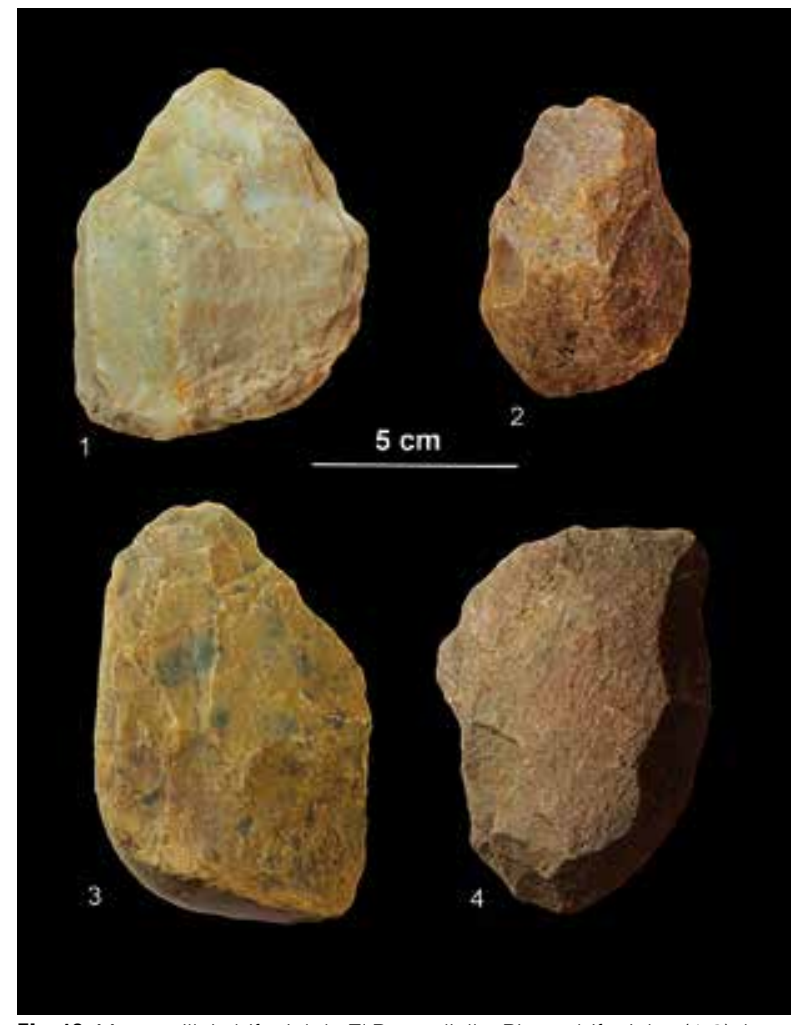

Fig. 19. Macroutillaje bifacial de El Barandiallu. Piezas bifaciales (1-3); hendedor (4). / Bifacial macrotool set from El Barandiallu. Bifacial pieces (1-3); Cleaver (4). (Fotos: J. Aspra)

En cuanto a los cantos trabajados, debemos destacar que también en otro yacimiento cercano adscrito al Paleolítico medio -igualmente al aire libre-, como es Bañugues, se ha descrito un importante conjunto de cantos tallados, para los que hemos propuesto distintos usos y clasificaciones (Álvarez-Alonso et al., 2014). La presencia de cantos trabajados en contextos del Paleolítico medio es algo relativamente común, y también se ha documentado en otros lugares como por ejemplo la cueva de Los Moros de Gabasa (Utrilla et al., 2014) o Lezetxiki (Álvarez-Alonso y Arrizabalaga, 2012). Por sus características, estas piezas están ligadas a funcionalidades similares a bifaces y hendedores (trabajo con madera, procesado de carcasas, o acceso al tuétano), por lo que pudieran ser piezas alternativas a éstas, ante posibles circunstancias derivadas de la escasez de materias primas para poder elaborarlas o fruto de la inmediatez en el uso y en la ocupación. En estos casos, y a diferencia de lo que se plantea en muchos contextos musterienses para los bifaces, con una vida útil más duradera (Soressi, 2002), los cantos trabajados serían piezas de uso y abandono inmediato, por tanto, no portables.

En lo que respecta a los bifaces y hendedores de El Barandiallu, están realizados sobre lascas grandes y medianas. Este tipo de formatos no han sido identificados en el conjunto, ni en el entorno del yacimiento, por lo que es probable que estén aportados en un soporte ya preparado o directamente elaborados, extremo que parece ser una constante en otros contextos musterienses con bifaces, de características similares, localizados en la Dordoña y el País Vasco francés (Brenet et al., 2017).

Los bifaces del conjunto son mayoritariamente de pequeño tamaño, en su mayoría espesos, presentando en varios casos retoque de raedera, con lo cual se asimilan con los tipos también denominados como bifaz-útil, tan frecuentes en muchos contextos musterienses sobre todo en el MTA (Soressi, 2002; Turq, 2000). Su morfología también sería una consecuencia directa del tipo y formas en que se encuentra la cuarcita local.

En lo relativo a los hendedores, se trata de piezas poco diagnósticas, aunque uno de ellos, de pequeñas dimensiones y retoque bifacial, es morfológica y tecnológicamente muy similar a los hendedores del nivel XIII basal de La Viña o Bañugues, también de cuarcita (Álvarez-Alonso, 2012; Álvarez-Alonso et al., 2014; Fortea, 1999). Esta convergencia lo hace especialmente interesante, ya que son pocas las piezas de este tipo contextualizadas estratigráficamente en el extremo occidental cantábrico. Otros casos más clásicos en la región, como El Castillo, El Pendo o Morín -o el yacimiento al aire libre de La Verde-, han aportado numerosas series líticas con un número elevado de hendedores, en sus niveles adscritos al Musteriense (Cabrera, 1984; González-Echegaray y Freeman, 1978; González-Echegaray et al., 1980; Montes, 2003). Los hendedores son piezas que, aunque ya aparecen de manera temprana en el Achelense peninsular, prolongan su presencia hasta momentos muy avanzados, siendo notable y característica su presencia en el Musteriense cantábrico (MIS 5e-3), hasta tal punto que esta circunstancia llegó a derivar en la definición de una facies propia, el "Vasconiense", muy discutida pero que recientemente ha vuelto a ser reconsiderada (Bordes, 1953, 1961; Freeman, 1966; Cabrera, 1983; Deschamps, 2009, 2017; Álvarez-Alonso et al., 2014).

En definitiva, la asociación presente en El Barandiallu entre macroutillaje y un abundante utillaje retocado sobre lasca (raederas y denticulados), con presencia limitada de algunas piezas tales como raspadores, buriles o perforadores, recuerda a los casos descritos para el MTA en Aquitania aunque, tipológicamente y siendo estrictos con las definiciones clásicas (Bordes, 1953, Soressi, 2002), los porcentajes presentes en El Barandiallu no permiten englobar el conjunto en ninguno de los dos subtipos descritos ( $\mathrm{A} \circ \mathrm{B}$ ). Por el contrario, desde un punto de vista tecnológico y observando el aspecto general del conjunto, esta vinculación sí parece bastante lógica.

\subsection{Síntesis de las Cadenas operativas líticas de El Barandiallu}

Hemos tratado de analizar este conjunto lítico, utilizando como unidad básica la descripción y compren- 
sión de las cadenas operativas líticas (Leroi-Gourhan, 1964; Pelegrin et al., 1988; Geneste, 1991; Boëda et al., 1990). De este modo, las cadenas operativas identificadas se articularían en torno a cinco fases o etapas que se integran dentro de los procesos de adquisición-producción-consumo: 1) captación/aprovisionamiento; 2) talla o debitado; 3) producción; 4) uso; 5) abandono/ amortización. Además, podemos incluir una sexta etapa, que se basaría en la reutilización de elementos previamente amortizados y abandonados, replicando las fases 2, 3 y 4, sobre piezas que ya se encuentran en la etapa 5. Esta reutilización de algunos elementos nos indica que el agregado lítico que hemos estudiado es posiblemente un producto de varias fases de actividad sucesivas en el tiempo.

Al menos se identifican tres grandes cadenas operativas, que luego a su vez se pueden estructurar y dividir en distintos procesos.

En primer lugar, una cadena operativa orientada a la producción de útiles muy determinados sobre soportes de sílex, que está incompleta y de la que solo tenemos elementos finales, algún resto de talla y algún fragmento de nódulo. Debido a su bajo número y al pertenecer a materiales diferentes, nos invita a pensar que se trata de retazos de otras cadenas operativas comenzadas en otro lugar y, fundamentalmente, se trataría de elementos finales transportados en ese estado, así como restos de reavivado o acondicionamiento.

En segundo lugar, existe una cadena operativa realizada íntegramente en cuarcita y cuyo objetivo es la producción de elementos de macroutillaje a partir de cantos o de grandes lascas.

Esta cadena no se encuentra completa, tratándose fundamentalmente de elementos en fase terminal, que seguramente han sido transportados en su estado final o en un grado de elaboración muy avanzado puesto que no hay evidencias de la talla de grandes nódulos y de la obtención de lascas de gran tamaño en el yacimiento. Algunas de las piezas, como los cantos tallados, sí han podido gestionarse en el yacimiento, al igual que sucede con la mayor parte de los núcleos donde su elaboración sí podría haberse llevado a cabo en el propio yacimiento.

En último lugar, nos encontramos con la cadena operativa más numerosa e íntegra, enteramente elaborada en cuarcitas locales. Esta cadena operativa está orientada a la obtención de soportes -lascas- de pequeño y mediano tamaño para su uso o conversión en distintos tipos de utillaje mediante retoque. La presencia de distintos tipos de núcleos (con intenso aprovechamiento), así como productos de avivado y acondicionamiento que reflejan diferentes fases de la preparación de los mismos, unido a un elevado número de productos que indican distintos grados de explotación, es síntoma de una importante actividad de talla. En este sentido, aunque se constata una variedad amplia y suficiente de córtex en los productos de talla, parece que algunas de las fases previas de la talla podrían haberse realizado en otros lugares. En consecuencia, esta cadena operativa en cuarcita parece haberse realizado prácticamente en su totalidad en el yacimiento, a excepción de algunas fases seguramente relacionadas con un desbastado previo.

Dentro de esta gran cadena operativa se emplean tanto cantos como fragmentos de canto y productos de talla con el fin de ser explotados como núcleos, para obtener lascas con filos cortantes y soportes para ser retocados, mediante el empleo de distintos métodos, siempre adaptados al soporte. Se trata de la cadena operativa más importante y la única que ha sido elaborada casi en su totalidad en el yacimiento implicando, seguramente, la obtención de un utillaje para un uso inmediato, como refleja también el elevado número de útiles ya amortizados existentes en el conjunto lítico.

En lo que respecta a los núcleos sobre canto, dentro de esta última cadena operativa se ha podido estructurar el aprovechamiento de cantos de cuarcita para su transformación en núcleos, a partir de tres modalidades que generan diferentes tipos de productos y dan pie para la implementación de los distintos métodos de talla presentes en el conjunto, como se puede observar en el esquema adjunto (Fig.20). En primer lugar, los cantos pueden ser utilizados directamente como percutores o gestionados en función de su morfología y tamaño para obtener distintos productos que, a su vez, sirven como soportes para obtener lascas mediante métodos de talla adaptados a cada morfología. En este segundo caso, como se observa en la figura 19, los cantos se fracturan de forma variada pudiendo, a partir de cada uno de los restos obtenidos, generar diferentes tipos de núcleo. Aunque no se expone en el esquema, las lascas corticales de mayor tamaño también son susceptibles de convertirse en núcleos de tipo unifacial o bifacial.

Finalmente, resulta curioso el hecho de encontrar algunos puntos en común con el conjunto lítico de Bañugues, puesto que, en este último y cercano yacimiento, también se registra un importante aprovechamiento de cantos rodados de cuarcita (Álvarez-Alonso et al., 2014: 9-12). Al igual que en Bañugues, en El Barandiallu también se han documentado los mismos tipos de percutor sobre canto tallado, núcleos NUPC y otros productos que confirman un tipo de explotación adaptada a los cantos rodados en el contexto del Musteriense local al aire libre. Esto puede ser fruto de una funcionalidad y tipo de gestión específica de la materia prima, en un contexto de mayor inmediatez y por lo tanto más versátil, que parece ser una característica en los yacimientos al aire libre con respecto a los sitios de ocupación en cueva de esta región.

En El Barandiallu se han descrito núcleos de tipo NUPC, percutores sobre canto tallado y cantos trabajados dentro de un contexto musteriense, dominado por denticulados y raederas, con muchas semejanzas con Bañugues, aunque con una diferencia notable con res- 


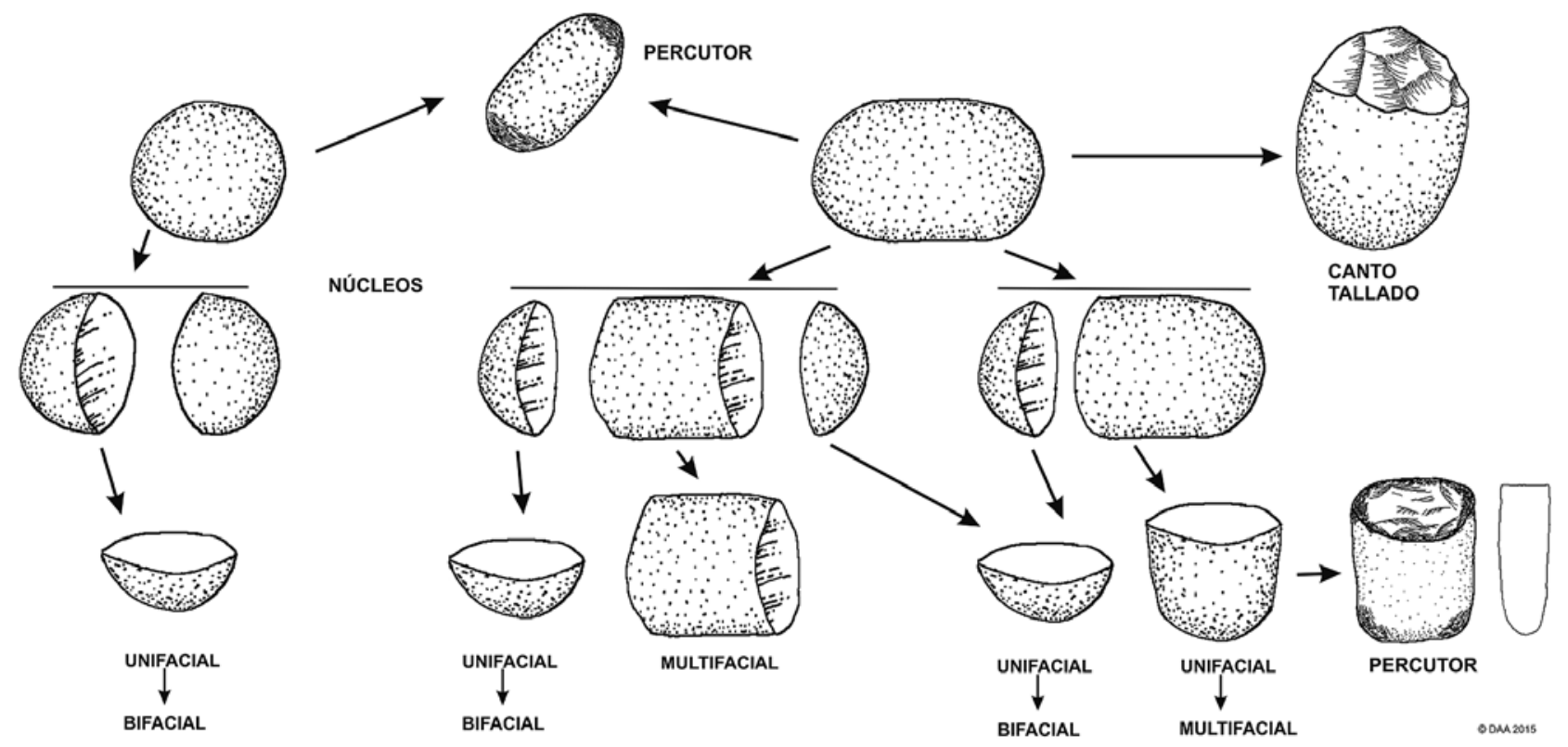

Fig. 20. Esquema de la cadena operativa identificada para el procesado de los cantos rodados y su conversión en núcleos, cantos tallados o percutores. / Outline of the identified chaîne opératoire for the knapping of the rounded pebbles and their conversion in cores, chopping tools or hammers.

pecto a los cantos de este último yacimiento, que al ser de mayor tamaño condicionan un aumento en la talla del macroutillaje (bifaces, hendedores, cantos tallados).

\section{DISCUSIÓN: ADAPTACIÓN Y VARIABILIDAD TECNOLÓGICA DE EL BARANDIALLU EN EL CONTEXTO DEL MUSTERIENSE CANTÁBRICO}

A la hora de analizar este conjunto lítico, debemos considerar la dependencia tecnológica que determina el uso mayoritario de unas materias primas esencialmente locales que podríamos definir como de extrema inmediatez en su captación. Esto, lógicamente condiciona los métodos de talla desarrollados y su imagen final, así como el aspecto de la composición tipológica resultante. Por otra parte, es innegable que estas estrategias de talla, así como los planteamientos tecnológicos que se desarrollan en El Barandiallu, responden a un contexto general que parece ser el de las poblaciones neandertales del Pleistoceno superior (MIS 4 y 3). Por eso mismo, a la hora de efectuar un intento de aproximación cronocultural exclusivamente a partir de la industria lítica, debemos ser conscientes del grado de adaptación tecnológica presente en el conjunto, pero también de la variabilidad existente en un contexto mucho más amplio, que incluso excede a la propia región cantábrica.

De esto modo, intentaremos resumir las características adaptativas percibidas en la industria de El Barandiallu y su grado de variabilidad en relación al contexto en el que creemos que debe enmarcarse este conjunto lítico.

\subsection{La adaptación tecnológica en el caso de EI Barandiallu}

A partir de las materias primas y de los núcleos empleados para llevar a cabo los distintos métodos de explotación lítica, y tras el análisis de los métodos de talla y las cadenas operativas presentado en el punto anterior, podemos deducir varias cuestiones, pero sin duda el aspecto más significativo es el empleo sistemático de cantos de cuarcita. Hemos observado su aprovechamiento intensivo, no desechándose ninguno de los productos y restos generados tras su fractura, ya que algunos de ellos serán transformados nuevamente en núcleos.

En primer lugar, hemos comprobado cómo en El Barandiallu se utilizan generalmente superficies lisas y corticales como plataformas de impacto y, en menos ocasiones, se llegan a preparar estas mediante un facetado parcial de dichas; en este último caso, estos núcleos no tienen por qué estar jerarquizados en su totalidad, pudiendo limitarse la preparación de superficies a zonas muy concretas del mismo, e incluso a la extracción de algunas lascas ya en las últimas etapas de explotación. Esta preparación o facetado de las superficies de impacto parece ser un recurso utilizado ante la necesidad específica de buscar un punto de impacto determinado para continuar explotando el núcleo, pero no representa una estrategia generalizada y recurrente dentro del conjunto.

También hemos observado que esta preparación de plataformas es un recurso que a veces se combina en un mismo núcleo con la extracción de lascas so- 
bre superficies lisas o corticales. Esto parece indicar que la jerarquización, la preparación de superficies de impacto (característica, por ejemplo, en los núcleos Levallois), es un recurso conocido, pero que únicamente se emplea como una solución técnica adaptada a las circunstancias de cada caso: en función del volumen, morfología o relaciones angulares de cada una de las matrices; para fines muy concretos, pero no como una estrategia sistemática.

Por esta razón, tanto los núcleos como los productos resultantes son muy variados y altamente versátiles, llegando incluso a resultar difícil su encuadre en esquemas concretos. Igualmente, consideramos que los distintos porcentajes de métodos de explotación presentes son, en este caso, un ejemplo nítido de adaptación técnica. Así, dentro de unos esquemas tecnológicos que contemplan distintas variables y posibilidades, en El Barandiallu se opta por recursos técnicos que se adaptan a las características de la materia prima mayoritaria, variando a lo largo de cada cadena operativa e incluso combinando diferentes técnicas en el mismo proceso, a medida que la morfología de cada núcleo se va modificando. Sin duda, se trata de un ejemplo muy claro de variabilidad tecnológica dentro del Musteriense, así como de la versatilidad técnica existente en este momento.

Los esquemas Levallois, discoide o Quina se presentan de forma muy clara en la región cantábrica, en la mayor parte de ocasiones (Carrión y Baena, 2003; Carrión et al., 2008; Ríos, 2008, 2012), aunque en algunos yacimientos al aire libre con conjuntos líticos reducidos que, seguramente, responden a necesidades más inmediatas y actividades menos planificadas y recurrentes, aparecen a veces de manera difusa o poco nítida. El resultado son conjuntos líticos como El Barandiallu, donde se perciben esquemas Levallois y discoides muy nítidamente, mientras que, en una parte importante del conjunto parecen fundirse, conjugarse o intercambiarse estos planteamientos tecnológicos en las mismas piezas. Un ejemplo de esto sería la preparación de plataformas de percusión, que da como resultado el facetado de los talones y la existencia parcial de planos jerarquizados en algunos núcleos discoides, o la conversión de núcleos Levallois en discoides a medida que se avanza en su explotación. En este caso, la adaptación o versatilidad del método de talla al tipo y morfología de la materia prima es muy evidente.

Esta circunstancia parece ser una pauta bastante habitual de adaptación a la materia prima, algo que en los conjuntos en cueva puede ser un condicionante menor, o no tan acentuado, puesto que las estrategias de talla y explotación son más complejas o completas que en los conjuntos cantábricos al aire libre.

\subsection{El Barandiallu en el contexto de las facies musterienses}

La asociación de un conjunto lítico numeroso dominado por denticulados y raederas, donde hay presencia minoritaria de hendedores y bifaces, encaja con una serie de niveles y conjuntos musterienses del norte peninsular: La Viña XIII basal, El Castillo 20, Morín 16-13, Lezetxiki V o Abauntz h (Álvarez-Alonso, 2012, 2014; Álvarez-Alonso et al., 2014). Para algunos de estos niveles se ha llegado a barajar una adscripción dentro del Musteriense de Tradición Achelense -MTAo a la controvertida facies Vasconiense (Benito, 1981; Mazo et al., 2011-12), mientras que en otras ocasiones se aceptó la imposibilidad de encajar estos niveles en las facies musterienses propuestas, proponiendo alternativas como "Musteriense final con hendedores" ante la irrelevancia apreciada en este debate tipológico (Fortea, 1999: 40), o directamente a un Musteriense típico con hendedores (Cabrera y Neira, 1994).

En nuestra opinión, y siendo estrictos con los postulados tipológicos y las facies musterienses clásicas, ninguno de los niveles cantábricos -y El Barandiallu no es una excepción- encajan exactamente en la definición tipológica del MTA (Bordes, 1953, 1961; Soressi, 2002). Por otra parte, la discutida existencia del Vasconiense cantábrico (Freeman, 1966; Cabrera, 1983) tampoco parece ser una explicación por sí misma a estos conjuntos con bifaces y hendedores, a pesar de recientes reinterpretaciones para el ámbito aquitano y pirenaico (Deschamps, 2009, 2017).

Pero, a pesar de esto y sin dejar de ser conscientes que las definiciones y clasificaciones tipológicas presentan serios problemas cuando se sale del ámbito en el que fueron planteados, sí es cierto que existen ciertas afinidades entre algunos de estos tecnocomplejos cantábricos, con el MTA. Esta circunstancia nos hace buscar una explicación convincente a la convergencia de determinadas características de algunos conjuntos musterienses cantábricos posteriores al MIS 5e con el MTA, que bien pudiera ser reflejo de una adaptación funcional de las poblaciones neandertales cantábricas, bien como una influencia tecnológica ${ }^{3}$ entre poblaciones vecinas o directamente como una adaptación de los mismos grupos humanos a condiciones y materias primas distintas.

En cualquier caso, parece claro que, tanto el MTA de Aquitania, como el fenómeno cantábrico que ha sido definido en algunos casos como Vasconiense, en otros como un MTA o simplemente como un Musteriense con macroutillaje (bifaces o hendedores), podrían estar vinculados estrechamente, siendo las diferencias

\footnotetext{
${ }^{3}$ Por el establecimiento de vínculos entre poblaciones cantábricas y aquitanas, o directamente por la movilidad de poblaciones aquitanas hacia la región cantábrica, con la consecuente adaptación de sus tradiciones tecnológicas culturales a la materia prima existente a lo largo del Cantábrico.
} 
percibidas fruto de la existencia de materias primas diferentes con menor calidad en el Cantábrico -sobre todo en cuanto al sílex-, de aspectos adaptativos o incluso de cuestiones funcionales. En primer lugar, pudiera parecer que es difícil creer que dos fenómenos tan cercanos y coetáneos muestren elementos similares y tan característicos como los aquí presentes, sin existir algún tipo de vínculo o relación. Este argumento debe cobrar fuerza ante la evidencia de los estudios paleoantropológicos y genéticos que demuestran una baja variabilidad genética en las poblaciones del occidente europeo, acompañado de una alta movilidad subsistencial, lo cual nos podría hacer pensar en una misma población o grupo poblacional tanto para las ocupaciones humanas en Aquitania como para el occidente pirenaico y la región cantábrica; áreas todas ellas que comparten este tipo de casuísticas (Lalueza et al. 2006; Rasilla et al., 2011).

Una de las principales diferencias entre Aquitania y la región cantábrica, es la mayor abundancia de sílex de buena calidad en la primera y, sobre todo, en formatos de mayor tamaño. Esto, seguramente ha favorecido que gran parte del macro-utillaje en esta zona haya podido ser elaborado en sílex (bifaces) mientras que, en el Cantábrico, es más raro -aunque hay ejemplos de bifaces que se asemejan a los del MTA francés (Arrizabalaga, 1994)-, siendo el macroutillaje, fabricado fundamentalmente en cuarcita. Por otra parte, estas dos regiones comparten una característica, que en ambos casos los hendedores son preferentemente de cuarcita u ofita. En el caso del MTA francés, los bifaces de sílex bien elaborados y en materiales que son ajenos al entorno del yacimiento, suelen proceder de otras localizaciones que rara vez exceden los $50 \mathrm{~km}$ de distancia (Soressi, 2002); es decir, hay movilidad de estas piezas, pero en un ámbito territorial muy bien definido, y no parece que a largas distancias. La traslación de los esquemas tecnológicos de las poblaciones neandertales aquitanas al Cantábrico, pudieron sufrir una adaptación por el imperativo de las materias primas, resultando tecnocomplejos "afines" al MTA pero que escapan a las definiciones tipológicas clásicas creadas a partir del registro francés.

En sitios como Le Moustier G, La Rochette, La Grotte XVIc, que presentan conjuntos asignados al MTA, caracterizados por el dominio de materias primas locales (donde las materias primas foráneas nunca sobrepasan el 3\% y las distancias de aprovisionamiento más largas son de $50 \mathrm{~km}$ ), más del 25\% del utillaje sobre lasca son raederas, con un número abundante de denticulados y escotaduras y con una proporción variable de útiles del Paleolítico superior (Soressi, 2002). Entre el MTA-A y el MTA-B apenas hay diferencias tecnológicas sustanciales, con métodos de talla similares; tan solo se aprecian diferencias entre ambos conjuntos en la organización de las actividades de talla y en que el MTA-A es ligeramente anterior y perdura algo más el MTA-B.
Por otra parte, es interesante destacar que en contextos musterienses con presencia de tipos diferentes de macroutillaje (cantos trabajados, bifaces y hendedores) se ha barajado, a partir de estudios traceológicos y también experimentales, una posible diversidad funcional de los mismos como explicación a esta variedad. De este modo, E. Claud $(2008,2012)$ mencionó para varios yacimientos del MTA, la preferencia del uso de bifaces en labores relacionadas con el procesado cárnico, mientras que los hendedores estarían más ligados a un uso con madera, extremo también compartido por otros estudios en yacimientos peninsulares (Utrilla y Mazo, 1996; Utrilla et al., 2014), aunque no parece que se deba a las mejores cualidades de la cuarcita con respecto al sílex, ya que este último se ha mostrado mucho más eficaz en esta tarea, según las experimentaciones realizadas (Domingo, 2013). Estudios más recientes sobre los hendedores asociados al denominado "Vasconiense", entre los que se encuentra el nivel 20 de El Castillo, han puesto de manifiesto que estos hendedores en cuarcita del Musteriense final franco-cantábrico, han sido probablemente usados, tanto con madera como en labores de carnicería en el procesado de carcasas (Claud et al., 2015), no arrojando así demasiada luz a la preferencia por este tipo de piezas en el Musteriense local con respecto a los bifaces.

Aunque el MTA no aparezca de forma nítida y clara al sur de los Pirineos y a lo largo del Cantábrico, no parece descabellado plantear una estrecha vinculación de unos y otros tecnocomplejos a ambos lados de los Pirineos, sobre todo entre Aquitania y la región cantábrica. Las resonancias de un MTA desdibujado, o los criterios que llevaron a hablar del Vasconiense, si bien no pueden ser defendidos desde un punto de vista tipológico, sí responden a tendencias tecnológicas y a patrones que pueden llegar a ser equiparados. Las materias primas imprimen una personalidad concreta a las industrias líticas, lo cual es reflejo de la capacidad de adaptación y variabilidad de los conocimientos y destrezas técnicas, así como de la tradición tecnológica de los grupos neandertales entre los MIS 4 y 3.

En definitiva, lo que podría ser una especialización para bifaces y hendedores en el MTA del suroeste francés (tanto en su uso como en su fabricación), donde el sílex es más abundante y de mejor calidad (y, sobre todo, de mayor tamaño) podría haberse convertido en la región cantábrica en una pérdida de dicha especialización, con un aumento de los hendedores y continuando con la presencia de bifaces, aunque ahora también fabricados en la misma materia prima.

En lo que respecta a la concepción de las distintas "facies musterienses", queda demostrado que las cronologías son las mismas, y muchas de las características son similares entre los distintos niveles peninsulares asignados a cada uno de los grupos. Esto ha roto hace mucho tiempo con este planteamiento de facies, ya que la variabilidad existente en el seno del Musteriense 
es incompatible con esos rígidos esquemas, nacidos al calor de una serie de conjuntos muy específicos en Francia y que se han demostrado insostenibles, para muchos yacimientos peninsulares (Rasilla y Santamaría, 2013). No obstante, creemos que eso no implica que en algunos casos estas facies no puedan remitir a determinadas tradiciones tecnológicas, culturales y funcionales que pueden encontrar un paralelo más allá de la estricta norma de la composición tipológica.

\section{CONCLUSIONES}

En primer lugar, hay que destacar la homogeneidad del conjunto lítico de El Barandiallu, independientemente de su procedencia diversa (superficie y excavación) y del estado de conservación del yacimiento. Como ya hemos apuntado, al margen de los problemas tafonómicos existentes, el yacimiento presenta una gran integridad espacial y arqueológica, pudiendo determinarse que se trata de un conjunto in situ a pesar de las remociones producidas por el arado, con distintas cadenas operativas en distinto grado de explotación y gestión, que han podido ser bien identificadas. Se trata de uno de los conjuntos musterienses al aire libre en la región cantábrica, más amplios y mejor caracterizados. Igualmente, es el único caso estudiado en el norte peninsular para un conjunto de este tipo en el que se ha identificado un proceso tafonómico provocado por la acción agrícola moderna y contemporánea.

En cuanto al conjunto lítico se refiere, en este caso se ha podido analizar la importancia de la materia prima, tanto en su calidad como en su morfología, como condicionante para el proceso tecnológico llevado a cabo por los neandertales en El Barandiallu. En definitiva, este asentamiento parece ajustarse a las características de una ocupación temporal, con un grado de inmediatez en la captación de recursos (la mayor parte de la materia prima es local) y en las cadenas operativas desarrolladas bastante elevado, aunque se ha podido identificar una gestión diferente en función de la cadena operativa, tanto a nivel de materia prima como a nivel tecnológico. Además, también se han podido observar la existencia de cadenas operativas íntegras y amortizadas que apuntalan esa inmediatez de la que hablamos (son la mayoría), a la vez que se ha determinado el transporte de piezas desde otros lugares, relativamente cercanos (macroutillaje) o más lejanos (piezas de sílex).

De este modo, podemos afirmar que en el caso de El Barandiallu la variabilidad tecnológica percibida en este conjunto puede ser una consecuencia del tipo de funcionalidades y circunstancias de la ocupación humana al aire libre, con respecto a las ocupaciones en cueva, más recurrentes y menos efímeras. Este asentamiento, podría corresponderse con el área de captación y espacio territorial para otros yacimientos en cueva cercanos, como podría ser La Viña, pudiendo trazarse conexiones a partir de las materias primas, tanto con la cuenca del Nalón como con la del Sella.
Por otra parte, a la hora de buscar un paralelo para El Barandiallu, resulta ineludible fijarse en el suroeste francés, donde se localizan varios yacimientos musterienses ubicados entre el final del MIS 4 y el MIS 3, en los que se documentan conjuntos con presencia de talla Levallois y discoide con un discreto porcentaje de bifaces (en sílex no local) y, en el menor de los casos, de algunos ejemplares de hendedores realizados, a diferencia de los bifaces, en cuarcitas u ofitas locales (Brenat et al., 2016). Especialmente nos interesan yacimientos como La Prissé, Chemin de Jupiter o Latrote, todos ellos al aire libre, donde se localizan hendedores sobre lasca en cuarcita y algunos bifaces en sílex de procedencia lejana, en un contexto donde las materias primas son fundamentalmente locales (Brenat et al., 2016).

A nivel más inmediato, es posible que la cronología relativa de El Barandiallu, con una caracterización de tecnotipológica que hemos hecho compatible con los contextos del MTA aquitano se ajuste con la de estos yacimientos franceses, así como también podamos establecer un vínculo con conjuntos en cueva como Abauntz h, La Viña XIII basal, identificado como un nivel del Musteriense final con hendedores, El Castillo 20, o las series musterienses con hendedores de Cueva Morín. Estos paralelos nos remiten a un contexto relativamente reciente dentro del Musteriense para El Barandiallu, que situamos en todo caso como contemporáneo o incluso posterior al MIS 4. Otras características del conjunto, como la identificación de esquemas operativos técnicos que podemos englobar dentro del concepto Quina (aunque no haya sido identificado este tipo de retoque), apuntalaría también una cronología reciente, a la vista del contexto cronológico peninsular para este tipo de manifestaciones musterienses (Carrión y Baena, 2003).

\section{AGRADECIMIENTOS}

La excavación de El Barandiallu contó con la financiación del Ayuntamiento de Llanera y la empresa Fluor S.L. A María de Andrés Herrero.

\section{REFERENCIAS BIBLIOGRÁFICAS}

Altuna, J., Baldeón, A., Mariezkurrena, K., 1990. La cueva de Amalda (Zestoa, País Vasco). Ocupaciones paleolíticas y postpaleolíticas. Colección Barandiarán 4. Eusko lkas kuntza, San Sebastián.

Álvarez-Alonso, D., 2012. El primer poblamiento humano en la región cantábrica. Reflexiones y síntesis en torno al Paleolítico antiguo. Kobie, 31, 21-44

Álvarez-Alonso, D., 2013. El Paleolítico en la cuenca del río Aboño (Llanera). Excavaciones en los yacimientos de El Barandiallu y la cueva del Olivo. En: Excavaciones Arqueológicas en Asturias 2007-2012, 57-68. Consejería de Cultura, Principado de Asturias.

Álvarez-Alonso, D., 2014. First Neanderthal settlements in northern Iberia: The Acheulean and the emergence of Mousterian technology in the Cantabrian region. Quaternary International 326-327, 288-306. 
Álvarez-Alonso, D., 2015. Los yacimientos arqueológicos en contextos edáficos superficiales. El caso del Paleolítico inferior y medio del norte de la Península Ibérica (España). Nailos,Estudios Interdisciplinares de Arqueología 2, 17-47.

Álvarez-Alonso, D., Arrizabalaga, A., 2012. La secuencia estratigráfica inferior de la cueva de Lezetxiki (Arrasate, País Vasco). Una reflexión necesaria. Zephyrus LXIX, 15-39.

Álvarez-Alonso, D., Rodríguez Asensio, J. A., Jordá Pardo, J.F., 2014. Reflexiones en torno a la caracterización tecnotipológica del yacimiento de Bañugues (Asturias, España) en el marco del Paleolítico medio del norte de la Península Ibérica. Munibe Antropologia-Arkeologia 65, 5-24.

Arbizu, M., Arsuaga, J.L., Adán, G.E., 2005. La cueva del Forno/Conde (Tuñón, Asturias): un yacimiento del tránsito del paleolítico medio y superior en la Cornisa Cantábrica. In: Lasheras, J.A., Montes, R. (eds.), Neandertales Cantábricos, estado de la cuestión, 10-38. Monografías del Museo de Altamira 20.

Arias, P., 1991. Estrategias de aprovechamiento de las materias primas líticas en la costa oriental de Asturias (VIII-III milenios A.C.). Treballs d'Arqueología 1 (Tecnología y Cadenas Operativas Líticas), 37-55.

Arrizabalaga, A., 1994. Hallazgo de un bifaz y otros restos líticos en el monte Jaizkibel (Hondarribia, Gipuzkoa). Munibe Antropologia-Arkeologia 46, 23-31.

Arrizabalaga, A., 2006. Lezetxiki (Arrasate, País Vasco). Nuevas preguntas acerca de un antiguo yacimiento. In: Cabrera, V., Bernaldo de Quirós, F., Maillo, J.M. (eds), En el centenario de la cueva de El Castillo: El ocaso de los Neandertales, 291309. Santander.

Arrizabalaga, A., Altuna J., Areso, P., falguères, Ch., Iriarte, $M^{\mathrm{a}}$. J., Mariezkurrena, K., Pemán, E., Ruiz, M., Tarriño, A., Uriz, A., Vallverdú, J., 2005. Retorno a Lezetxiki (Arrasate, País Vasco): Nuevas perspectivas de la investigación. In: Santonja, M., Pérez-González, A., Machado, M.J. (eds), Geomorfología y conservación del patrimonio, 63-80. Almazán (Soria).

Arrizabalaga, A., Ríos, J., Álvarez-Alonso, D., 2015. The Past is Out There. Open-air Palaeolithic sites and new research strategies in the Cantabrian region (northern Iberia). Quaternary International 364, 181-187.

Baena, J., Carrión, E., 2014. La cueva del Esquilléu como nuevo referente para el Musteriense cantábrico. In: Sala, R. (ed.): Los cazadores recolectores del Pleistoceno y del Holoceno en Iberia y el Estrecho de Gibraltar: estado actual del conocimiento del registro arqueológico, 82-87.

Benito del Rey, L., 1981. Aspectos técnicos y tipológicos que relacionan estrechamente el Muteriense con hendidores de las cuevas del Castillo y Morín (Santander). Munibe 33 (3-4), 157-170.

Bischoff, J. L., García, J. F., Straus, L. G., 1992. Uranium-series Isochron Dating at El Castillo Cave (Cantabria, Spain): The Acheulean/Mousterian question. Journal of Archaeological Science 19(1), 46-92.

Boëda, E., 1993. Le débitage discoïde et le débitage levallois récurrent centripète. Bulletin de la Societé Préhistorique Française 90(6), 392-404.

Boëda, E., 2006. Levallois: uma construçao volumétrica, varios métodos, uma técnica. Canindé, Revista do Museu de Arqueologia de Xingó 7, 37-78.

Boëda, E., Geneste, J.M., Meignen, L., 1990. Identification de chaines operatoires lithiques du Paleolithique Ancien et Moyen. Paléo 2, 43-70.

Bordes, F., 1953. Essai de Classification des industries moustériennes. Bulletin de la Societé Préhistorique Française 50, 457-466.
Bordes, F., 1961. Typologie du Paléolithique ancien et moyen. Publications de l'Institut de Préhistoire de l'Université de Bordeaux, mém. n. ${ }^{\circ} 1,2$ vol.

Bourguignon, L., Delagnes, A., Meignen, L., 2006. Systèmes de production lithique, gestion des outillages et territories au Paléolithique moyen: où se trove la complexité?. In: Astruc, L. Bon, F., Léa, V., Milcent, P.-Y., Philibert, S., Normes techniques et pratiques sociales. De la simplicité des outillages Pré- et Protohistoriques XXVle rencontres internationales d'archéologie et d'histoire d'Antibes, 75-86.

Brenet, M., Chadelle, J.P., Claud, E., Colonge, D., Delagnes, A., Deschamps, M., Folgado, M., Gravina, B., Ihuel, E., 2017. The function and role of bifaces in the Late Middle Paleolithic of southwestern France: Examples from the Charente and Dordogne to the Basque Country. Quaternary International 428, 151-169.

Cabrera, V., 1983. Notas sobre el Musteriense cantábrico: el "Vasconiense". Homenaje al prof. Martín Almagro Basch 1, 131-141.

Cabrera, V., 1984. La cueva de El Castillo (Puente Viesgo, Santander). Bibliotheca Praehistorica Hispana, vol. XXII. CSIC, Madrid.

Cabrera, V., Bernaldo de Quirós, F., 2000. Excavaciones arqueológicas en la cueva de El Castillo (Puente Viesgo). Actuaciones Arqueológicas en Cantabria 1984-1999, 23-32.

Cabrera, V., Neira, A., 1984. Los conjuntos líticos del Paleolítico medio cantábrico según el análisis de componentes principales. En: Lasheras, J.A. (ed.), Homenaje al Dr. Joaquín González Echegaray. Museo y Centro de Investigación de Altamira $17,55-60$

Carrión, E., Baena, J., 1998. Breves anotaciones sobre la colección musteriense de la cueva de Las Monedas (Puente Viesgo, Cantabria), depositada en el Museo de Prehistoria y Arqueología de Santander. CuPAUAM, Cuadernos de Prehistoria y Arqueología 25, 71-85.

Carrión, E., Baena, J., 2003. La producción Quina del nivel XI de la cueva del Esquilléu: una gestión especializada de la producción. Trabajos de Prehistoria 60(1), 35-52.

Carrión, E., Baena, J., 2005. El Habario: Una ocupación musteriense al aire libre en los Picos de Europa. In Montes, R., Lasheras, J.A. (eds.), Neandertales Cantábricos. Estado de la cuestión. Monografías del Museo Nacional y Centro de Investigación de Altamira 20, 443-460.

Carrión, E., Baena, J., Conde, C., Cuartero, F., Roca, M., 2008. Variabilidad tecnológica en el Musteriense de Cantabria. Treballs d'Arqueologia 14, 279-318.

Castanedo, I., 2001. Adquisición y aprovechamiento de los recursos líticos en la cueva de La Flecha. Munibe 53, 3-18.

Claud, E., 2008. Le statut fonctionnel des bifaces au Paléolithique moyen récent dans le Sud-Ouest de la France (Thèse de Doctorat). In: Étude tracéologique intégrée des outillages des sites de La Graulet, La Conne de Bergerac, Combre Brune 2, Fonseigner et Chez-Pinaud/Jonzac. Université de Bourdeaux 1.

Claud, E., 2012. Les bifaces: des outils polyfonctionnels? Étude tracéologique integrée de bifaces du Paléolithique moyen récent dans le Sud-Ouest de la France. Bulletin de la Société préhistorique française 109(3), 413-439.

Claud, E., Deschamps, M., Colonge, D., Mourre, V., Thiébaut, C., 2015. Experimental and functional analysis of late Middle Palaeolithic flake cleavers from southwestern Europe (France and Spain). Journal of Archaeological Science 61, 1-23.

Deschamps, M., 2009. Le Vasconien: révision de sa signification à partir des industries lithiques d'Olha I et II,d'Isturitz et de Gatzarria. Paléo 21, 103-126. 
Deschamps, M., 2017. Late Middle Palaeolithic assemblages with flake cleavers in the western Pyrenees: The Vasconian reconsidered. Quaternary International 433, 33-49.

Díez Martín, F., Sánchez Yustos, P., Gómez, J. A., Gómez de la Rúa, D., Yravedra, J., Díaz Muñoz, I., 2011. La ocupación neandertal en el Cañón de la Horadada (Mave, Palencia, España): Nuevas perspectivas arqueológicas en Cueva Corazón. Munibe Antropologia-Arkeologia 62, 65-85.

Domingo, R., 2013. La aptitud de los hendedores para el corte de madera: diferencias entre el sílex y la cuarcita. In: Palomo A., Terradas, X. (ed.), Experimentación en arqueología. Estudio y difusión del pasado, 265-269.

Estrada, R., Jordá Pardo, J.F., 2004. Arqueología y gas natural: el Paleolítico medio de El Barandiallu (Villabona, Llanera, Asturias central). Actas de la XI Reunión nacional de Cuaternario (2, 3 y 4 de Julio 2003, Oviedo), 253-260.

Falguères, Ch., Yokoyama, Y., Arrizabalaga, A., 2006. La geocronología del yacimiento pleistocénico de Lezetxiki (Arrasate, País Vasco). Crítica de las dataciones existentes y algunas nuevas aportaciones. Munibe Antropologia-Arkeologia 57(II), 93-106.

Freeman, L.G., 1966. The nature of Mousterian facies in Cantabrian Spain. American Anthropologist 68 (2), 230-237.

Fortea Pérez, J., 1999. Abrigo de La Viña. Informe y primera valoración de las campañas de 1995 a 1998. Excavaciones Arqueológicas en Asturias 1995-98, 31-41.

Geneste, J.M., 1991. Systèmes techniques de production lithique: variatons technoécnomiques dans les processus de réalisation des outillages paléolithiques. Techniques et culture 17-18, 1-35.

González Echegaray, J., Freeman, L.G., 1978. Vida y muerte en Cueva Morín. Institución Cultural de Cantabria.

González Echegaray, J., Freeman, L. G., Barandiarán, I., ApeIlaniz, J. M., Butzer, K. W., Fuentes Vidarte, C., Madariaga, B., González Morales, A., Leroi-Gourhan, A., 1980. El yacimiento de la cueva de "El Pendo" (excavaciones 1953-57). Biblioteca Praehistorica Hispana XVII. CSIC.

González Urquiijo, J.E., Ibáñez Estevez, J.J., Ríos Garaizar, J., Bourguignon, L., 2006. Aportes de las nuevas excavaciones en Axlor sobre el final del Paleolítico Medio. En: Cabrera Valdés, V., Bernaldo de Quirós, F., Maílo Fernández, J.M. (eds.), En el centenario de la cueva de El Castillo: el ocaso de los Neandertales, 269-289. Centro Asociado a la UNED-Cantabria, Santander.

Lalueza-Fox, C., Krause, J., Caramelli, D., Catalano, G., Milani, L., Sampietro, M.L., Calafell, F., Martínez-Maza, C., Bastir, M., García-Tabernero, A., Rasilla, M. de la, Fortea, J., Pääbo, S., Bertranpetit, J., Rosas, A., 2006. Mitochondrial DNA of an Iberian Neandertal suggests a population affinity with other European Neandertals. Current Biology 16(16), R630-R632.

Leroi-Gourhan, A., 1964. Le geste et la parole, I, Technique et langage. Albin Michel, Paris.

Maíllo, J.M., Arteaga, C., Iriarte-Chiapusso, Mª J., Fernández, A., Wood, R., Bernaldo de Quirós, F., 2014. Cueva Morín (ViIlanueva de Villaescusa, Cantabria). In: Sala, R. (ed.), Los cazadores recolectores del Pleistoceno y del Holoceno en Iberia y el Estrecho de Gibraltar: estado actual del conocimiento del registro arqueológico, 72-78.

Martín, P., Montes, R., Sanguino, J., 2006. La tecnología lítica del Musteriense final en la región cantábrica: los datos de Covalejos (Velo de Piélagos, Cantabria, España). In: Cabrera, V., Bernaldo de Quirós, F., Maíllo, J.M. (eds.), En el centenario de la cueva de El Castillo: El ocaso de los Neandertales, Centro Asociado a la UNED-Cantabria, 231-248.
Mazo, C., Utrilla, P., Blasco, F., Mandado, J., Torres, T., Ortiz, E., Rink, W. J. 2011-12. El nivel musteriense de la cueva de Abauntz (Arraitz, Navarra) y su aportación al debate "Vasconiense". Mainake XXXIII, 187-214.

Menéndez, M., Quesada, J.M., Jordá Pardo, J.F., Carral, P., Trancho, G.J., García Sánchez, E., Álvarez-Alonso, D., Rojo, J., Woods, R., 2009. Excavaciones arqueológicas en la cueva de La Güelga (Cangas de Onís, Asturias). Excavaciones Arqueológicas en Asturias 2003-2006, 197-209.

Menéndez, M., Weniger, G-CH., Álvarez Alonso, D., Andrés Herrero, M. de, García Sánchez, E., Jordá Pardo, J.F., Kehl, M., Rojo, J., Quesada, J.M. y Schmidt, I., 2014. Cueva de La Güelga. In: Sala, R. (ed.): Los cazadores recolectores del Pleistoceno y del Holoceno en Iberia y el Estrecho de Gibraltar: estado actual del conocimiento del registro arqueológico, 60-63.

Montes, R., 1999. El yacimiento al aire libre de El Hondal (Requejada de Polanco). Actuaciones arqueológicas y resultados gloarqueológicos. Sautuola VI (Homenaje al Dr. M. A. García Guinea), 35-44.

Montes, R., 2003. El primer poblamiento de la Región cantábrica. El Paleolítico inferior cantábrico. Museo Nacional y Centro de Investigación de Altamira, n 18. Ministerio de Educación, Cultura y Deporte, Madrid.

Montes, R., 2014. La secuencia estratigráfica de la cueva de El Pendo (Escobedo de Camargo, Cantabria). In: Sala, R. (ed.), Los cazadores recolectores del Pleistoceno y del Holoceno en Iberia y el Estrecho de Gibraltar: estado actual del conocimiento del registro arqueológico, 87- 91.

Pelegrin, J., Karlin, C., Bodu, P., 1988. «Chaînes opératories»: un outil pour le préhistorien. Technologie préhistorique, 55-62.

Rasilla, M. de la, Rosas, A., Cañaveras, J. C., Lalueza-Fox, C. (eds.)., 2011. La cueva de El Sidrón (Borines, Piloña, Asturias): investigación interdisciplinar de un grupo neandertal. Excavaciones Arqueológicas en Asturias, Monografías 1. Oviedo.

Rasilla, M. de la., Santamaría, D., 2013. Are Bordes' Mousterian facies discrete groups in the Iberian Peninsula?. Munibe Antropologia-Arkeologia 64, 5-15.

Rasilla, M. de la, Rosas, A., Cañaveras, J. C. y Lalueza, C. (eds.)., 2011. La cueva de El Sidrón (Borines, Piloña, Asturias). Investigación interdisciplinar de un grupo neandertal. Ed. Trea, Principado de Asturias.

Ríos, J., 2008. Variabilidad tecnológica en el Paleolítico medio de los Pirineos occidentales: una expresión de las dinámicas históricas de las sociedades neandertales. Treballs d'arqueologia 14, 171-194.

Ríos, J., 2012. Industria lítica y sociedade del Paleolítico médio al superior em torno al golfo de Bizkaia. Publican ediciones (col. Tesis doctorales).

Ríos, J., 2013. Aranbaltza. Yacimiento paleolítico al aire libre. Arkeoikuska 2013, 175-179.

Ríos, J., Gárate, D., Iriarte, E., Cearreta, A., Iriarte-Chiapusso, M.J., 2010. Los yacimientos de Mendieta I y II (Sopelana, Bizkaia): Dos ocupaciones al aire libre del Paleolítico inferior y medio. Kobie 29, 7-18.

Ríos, J., Gárate, D., Gómez-Olivencia, A., Iriarte, E., Arceredillo, D., Iriarte-Chiapusso, M. J., García-Ibaibarriaga, N., García, A., Gutiérrez-Zugastl, I., Torres, T., Aranburu, A., Arriolabengoa, M., Bailón, S., Murelaga, X., Ordiales, A., Ortiz, J. E., Rofes, J., San-Pedro, Z., 2015. Short-term Neandertal occupations in the late Middle Pleistocene of Arlanpe (Lemoa, northern Iberian Peninsula). Comptes Rendus Palevol 14(3), 233-244.

Sáenz de Buruaga, A., Hoyos, M., Ormazábal, A., 1999. Cronoestratigrafía y paleoclimatología de los depósitos prehistóricos de la cueva de Arrillor (Araba, País Vasco). Munibe Antropologia-Arkeologia 51, 137-151. 
Sánchez Yustos, P., Díez Martín, F., 2015. Dancing to the rythms of the Pleistocene? Early Middle Palaeolithic population dynamics in NW Iberia (Duero basin and Cantabrian region). Quaternary Science Reviews 121, 75-88.

Santamaría, D., Rasilla, M. de la, Martínez, L., Tarriño, A., 2011. Las herramientas y su interpretación cultural y económica. In Rasilla, M. de la, Rosas, A., Cañaveras, J.C. y Lalueza-Fox, C. (eds.), La cueva de El Sidrón (Borines, Piloña, Asturias): investigación interdisciplinar de un grupo neandertal. Excavaciones Arqueológicas en Asturias, Monografías 1, 137-145.

Soressi, M., 2002. Le Moustérien de tradition acheuléenne du sud-ouest de la France. Discussion sur la signification du faciès à partir de l'étude comparée de quatre sites: Pech-deI'Azé I, Le Moustier, La Rochette et la Grotte XVI. Thèse de I'Université Bordeaux I.

Tapia, J., 2010. El depósito paleolítico de La Garma A: estratigrafía y aproximación taxonómica. Trabajo de Investigación de 3er ciclo, Departamento de Ciencias Históricas, Universidad de Cantabria (inédito).

Tarriño, A., Duarte, E., Santamaría, D., Martínez, L., Fernández, J., Suárez, P., Rodríguez, V., Forcelledo, E., Rasilla, M. de la.,
2013. El Sílex de Piloña. Caracterización de una nueva fuente de materia prima en la Prehistoria de Asturias. In : De la RasiIla, M., Fortea Pérez, F. Javier (coord.). Universitatis Ovetensis Magister. Estudios en Homenaje, 115-132. Ediciones de la Universidad de Ménsula Ediciones, Oviedo.

Terradas, X., 2003. Discoid flaking method: conception and technological variability. In: Peresani, M. (ed.), Discoid Lithic Technology. Advances and Implications, 19-31. BAR Int.Series, 1120, Oxford.

Thiébaut, C., Mourre, V., Chlard, P., Colonge, D., Coudenneau, A., Deschamps, M., Sacco-Sonador, A., 2012. Lithic technology of the final Mousterian on both sides of the Pyrenees. Quaternary International 247, 182-198.

Utrilla, P., Mazo, C., 1996. Non Flint raw materials in La Rioja: a tentative interpretation. Non Flint Stone tools and the Palaeolithic occupation of Iberian Peninsula. B.A.R. 649, 63-80.

Utrilla, P., Montes, L., Domingo, R., 2014. Grandes cantos trabajados de la cueva de Los Moros 1 de Gabasa (Huesca). In: Arnáez, J., González-Sampériz, P., Lasanta, T., Valero Garcés, B.L. (eds.), Geología, cambio ambiental y paisaje: Homenaje al profesor José María García Ruiz, 129-141. 